\title{
EMPIRICAL RELATIONSHIP BETWEEN CORPORATE INCOME TAX, GOVERNMENT REVENUE, AND EMPLOYMENT IN OECD COUNTRIES
}

\author{
Zoumpoulidis Vassilios, \\ International Hellenic University, \\ Department of Accounting and Finance, \\ Campus of Kavala, 65404 Greece,
}

\begin{abstract}
This paper applies the technique of correlation analysis to find out the impact of corporate tax on government revenue and employment in OECD countries. The findings of the study suggest that there is no relationship between corporate income tax, government revenue, and employment during 2000 and 2019.
\end{abstract}

Keywords: corporate tax, taxation, government revenue, employment, OECD countries.

\section{Introduction}

Corporate taxation is an important source of revenue for the government (Cobham \& Jansky, 2018). Moreover, taxation is also an effective instrument for promoting equity and addressing social and economic concerns (OECD, n.d.). Studies have empirically verified that reduction in corporate income tax reduces capital constraints on firms that encourage the formation of corporations with the resultant increased employment levels ina country (Chen et al., 2018).

The paper focuses on the impact of corporate taxation government revenues and employment levels in countries that belong to the Organization of Economic Cooperation and Development (OECD) group. In OECD countries, a non-linear relationship exists between economic growth and corporate tax burden (Bodgan \& Maria, 2017). Increasing the tax burden was found to have a detrimental effect on economic growth in OECD countries in the long run (Bodgan \& Maria, 2017). The effect on the economy of increasing the tax burden in countries belonging to the OECD group was negative when the surplus tax revenues were spent on unproductive expenditures (Connolly \& Li, 2016).

The objective of the paper is to quantify and empirically verify the impact of corporate income tax on government revenues and employment levels between the period 2000 and 2019. The innovation of the paper is that it combines the systematic literature review with the empirical analysis regarding the topic. Moreover, the study is first of a kind in that it empirically verifies and determines the magnitude of the relationship between corporate income tax, government revenues, and employment in OECD countries using correlation analysis.

\section{Literature Review}

Taxation is one of the most important source of revenue for the government (Shakibaei \& Ahmadinejad, 2016). Corporate income tax is taxation on the corporate sector as opposed to the non-corporate sector (Harberger, 1962). Bua et al. (1990) had examined 800 industrial firms during the period 1980-87 and found that a decrease in the corporate tax led have a positive long term impact in employment and company activities. But Bua et al. (1990) had also found that for some companies a decrease in corporate taxation can have a negative effect on employment. The results imply corporate tax cuts can have long and short term effects and that the effect of tax cuts will be positive in long term duration.

Gober and Burns (1997) had studied the relationship between economic indicators and tax structure in OECD countries. The findings of the study found inconsistent effect on government revenues. However, Korovly (2020) had found that tax regulation has an important influence on the social stability and economic development.

Another study by Strulik \& Siegfried (1999) posits that corporate tax cuts leads to increased employment. Strulik and Siegfried (1999) used a mathematical model to suggest that tax cuts leads to higher marginal returns to investment and employment due to which the employment rate increases. Moreover, Strulik and Siegfried (1999) also suggest that an increase in employment is negatively correlated to the debt to equity ratio. A reduction in tax rates according to Strulik and Siegfried (1999) results in lower debt to equity ratio that spurs the corporation to invest more resulting in increased employment rate.

Feld and Kirchgasssner (2001) had used econometric models to investigate the impact of corporate tax on Swiss firms between 1985 and 1997. The findings of Feld and Kirchgasssner (2001) study show that increase in corporate income tax reduces the employment levels in the region. The authors had also cited works of Bartik (1985), Papke (1991) and Hines (1996) that showed a negative impact between corporate income taxes and employment levels. The study by Feld and Kirchgasssner (2011) concludes that companies prefer locations where the tax burdens is lower leading to increased employment in the region. Arnold (2008) had examined the corporate tax structures in 21 OECD countries and concluded that the corporate tax had a negative impact on the economic output of a country.

An IMF (2002) report that studied effect of corporate taxation in the Slovak Republic had concluded that corporate tax cuts, increased profitability, and corporate restructuring resulted in an increase in the fixed investment. The 
report by IMF (2002) had also found that an decrease in tax, rising employment and wages, and redeeming the National Property Funds accelerated private consumption.

Persson and Tabellini (1994) had found that increase in corporate taxation leads to reduced growth. A study carried out by Priess and Spooner (2003) who concluded that corporate tax was negatively related to employment growth. An increase in corporate taxation leads to an increase in employment growth while a decrease in corporate taxation leads to a decrease in the corporate taxation. Similar conclusions were made by Harden et al. (2003) who found that high corporate taxation is inefficient as it does not reduce the loss in employment.

An opposite view is presented by Uhlig and Yannagawa (1996) who had suggested that higher corporate taxation leads to increased growth within the endogenous growth model. Uhlig and Yannagawa (1996) had used a novel model that related the taxation and economic output through the impact of the tax burden on the supply of public goods and services. According to Uhlig and Yannagawa (1996), there is a non-linear influence of corporate taxation on economic growth. The findings by Uhlig and Yannagawa (1996) also suggest and increase in compensation of employees in OECD countries with higher corporate taxes and a positive impact on growth. A study by Nam and Radulescu (2004) suggest that the reduction in corporate taxation will not lead to increased investment when the inflation rate is high. Inflation levels may be the reason that Uhlig and Yannagawa (1996) found that increase in taxation leads to increased growth. The study by Uhlig and Yannagawa (1996) did not account for control factors such as inflation that could affect the economic activity.

Corporate taxation has also been found to result in a decrease in the wage rate. A study by Hassett and Mathur (2015) found an inverse relationship between corporate taxation and wage rates. The findings of Hassett and Mathur (2015) show that 1 percent increase in the corporate taxation rate leads to a 0.5 percent decrease in the wage rate. Hassett and Mathur (2015) had come to the conclusion after analysing a panel of 66 countries for a period of 25 years. Similar conclusion was drawn by a study carried out by Peichel et al. (2013) regarding German business tax whereby a one euro increase in the annual tax rate leads to a 50 cent decrease in the wage bill. According to Peichel et al. (2013), the burden of increase corporate tax is borne by high and medium skilled labor. The study by Piechel et al. (2013) had also found that the impact on the general equilibrium wage level in Germany was negligible due to high labor mobility.

However, another study carried out by Riedel (2011) had concluded that an increase in corporate taxation rate results in an increase in the wage level domestic workers and a decrease in wages of domestic workers. According to Riedel (2011), the increase in wages of domestic workers happen due to the worker's union wage bargaining as per the Separate Accounting (SA) model that is used in the EU region to tax corporate income. Riedel (2011) contends that the opposite happens under the formula apportionment (FA) method used in the US and Canada whereby the wages of the domestic workers decrease but the foreign workers increase upon increase in corporate tax rates. Moreover, a study by Dwenger et al. (2011) had found that any increase in the wages due to negotiation after a substantial corporate tax reform is negated due to a reduction in employment level.

Another study by Dwenger et al. (2011) had found that a decrease in the taxation by 1 euro results in an increase the wage bill by 0.47 euro. Dwenger et al. (2011) had made the conclusion after examining the effect of corporate taxation on employment in Germany between 1998 and 2006.But a study by Bajrami (2017) that had examined the effect of corporate taxation in Belgian had found that corporate taxation burden does not seem to be passed to the employees.

Corporate taxation affects firms within a country differently. Bernal et al. (2017) had found that an increase in the corporate taxation had positively affected employment in small and micro firms. Bernal et al. (2017) had examined formal employment data in non-public firms in Columbia and found that the effect of tax cuts on medium and large firms was negligible. Agarwal and Chakraborty (2019) had found that the effective corporate tax rate for small firms is generally higher than the tax rate for large corporations. The findings of the study by Agarwal and Chakraborty (2019) also found that the impact of the corporate taxation rate is more on the capital than on the labor.

Reduced taxation is found to lead to an increased investment (Ohrn, 2018). Lower taxation results in increased after-tax profit that enhances capacity of firms to invest in new technologies (Cai et al., 2018). Moreover, policy makers often use employment tax reduction as a public policy to stimulate economic recovery and growth (Zeli, 2020). A study of 19 member EU states founds that any changes in the fiscal and tax policy had an impact on the investments within a country (Mitroi, 2018). Studies have also found that countries with high wages generally charge a lower tax as compared to countries with lower wages (Mittermaier \& Rincke, 2013). It has been shown that countries compensate firms for the wage differential with an increase in compensation cost differential triggering a reduction in the corporate tax rate (Mittermaier \& Rincke, 2013). Moreover, studies have also shown that an increase in labor cost differential by one standard deviation leads to a decrease in the statutory corporate tax rate by 7.3 to 7.5 percentage points (Mittermaier \& Rincke, 2013).

A landmark study by economists at the OECD had found that the corporate taxation decrease the economic growth in a country (Hodge, 2016). A study by Kapko (2018) also found that an increase in corporate tax also resulted in reduced job creation. Andre and Hwang (2018) had examined firms in Finland and found that competitive corporate taxation resulted in increased local production. Using a dynamic stochastic model Chen et al. (2014) found that employment rate reduce the non-employment in a country by about 5.4 percent. Chen et al. (2014) study suggest that the 
employment is created due to reduced corporate taxation in the form of entry of new firms and existing firms changing the form of legal organization.

Sen and Cevik (2021) studied the effect of corporate tax rate in Turkey between the period 1980 and 2019. It was found that corporate income tax was negatively related to government corporate taxation revenues. Moreover, Sen and Kevik (2021) had also found that the revenue maximization rate is about 23.5 percent, which is near the statutory corporate taxation rate in Turkey of 22 percent. It was also concluded by Sen and Kevik (2021) that increasing taxation revenue through a hike in the corporate tax rate is not the right tax policy. According to Sen and Kevik (2021), a more effective strategy is to cut the corporate tax rate as it will have a positive effect on employment and economic growth.

Corporate taxation also has an effect on the capital mobility apart from trade within a country. Swank and Steinmo (2002) had carried out an empirical study examining 14 developed economies between the period 1981 and 1995. It was concluded by Swant and Steinmo (2002) that reduction in statutory corporate tax rates positively affects the trade and capital mobility in a country.

Studies show that the tax rates have been declining around the world (Kelly \& Graziani, 2004). Lowering the tax rates encourages investment that in turn leads to increased capital stock and productivity of employees (Kouparitas et al., 2016). Misuse of the taxation law does occur as companies carry on aggressive tax planning to reduce the tax burden through investment fund merger repetitions (Lukes \& Skalova, 2021). Some studies have also found that firms respond to tax cuts by increasing their physical capital and borrowings while remaining the employment levels unchanged (Li et al., 2021). In fact, studies have found that decreasing the corporate tax results in a decrease in the firm level labour share (Lie et al., 2021).

The literature review suggests that corporate taxation affects employment and growth. Our study aims to contribute to the literature review by using a Pearson correlation analysis to determine the impact of corporate taxation on employment and economic output in OECD countries. We intend to empirically confirm the existence of linear or non-linear relationship between corporate taxation, employment and government revenues in 36 OECD countries.

The remaining portion of the paper is structured as follows. Section 3 describes the hypothesis that will be tested in the study. Section 4 explains the data methodology used for analysing the data. Section 5 describes the results of the analysis and Chapter 6 concludes the study with a recommendation for future research.

\section{Hypothesis}

Previous studies offer mixed results about the impact of corporate taxations on employment levels in different countries. Some studies suggest that firms that pay less tax tend to create fewer employment opportunities (Leigh, 2018; Fuest et al., 2018). In contrast, another study posits that reducing corporate tax reduces the tax constraints that encourage the formation of $\mathrm{C}$ corporations resulting in increased employment (Chen et al., 2018). Moreover, a seminal paper by Harbenger (1962) cited by Chen et al. (2018) found no relationship between corporate tax and labour supply in a country. We are interested to find out whether there is a relationship between government taxation and unemployment rate in OECD countries, which leads to our first hypothesis.

H1: A relationship exists between corporate income taxation and unemployment rates in OECD countries.

According to the corporate-income tax rate-revenue paradox, corporate taxation causes tax revenues to increase but the GDP to fall (Dixon \& Nassios, 2006; Ohno, et al., 2015). We want to know if there actually exists any relationship between taxation and government revenues in a country.

H2: relationship exists between corporate income taxation and government revenues in OECD countries.

\section{Data Methodology \\ Data Analysis Method}

The Pearson correlation method has been used to find out the relationship between corporate income tax, government revenue and unemployment. The method has been selected to find out whether the variables selected for the study are related to each other. Our aim is to find out whether a change in one variable leads to a corresponding increase or decrease in another variable.

\section{Data Sample}

The data for the study were sourced from the OECD website. The OECD website provides current and historical economic data of all OECD countries, including taxation, government revenues in terms of gross domestic product (GDP), employment, public investment, government consumption, and many other metrics. The historical data regarding taxation, government revenues in terms of gross domestic product (GDP) has been reviewed for the study.

The sample consists of 34 OECD countries with data selected for the years 2000-2019. Table 1in Annexure shows the corporate income tax rates of selected OECD countries.

Table 2a in Annexure displays the GDP stats of 34 OECD countries between the period 2000 and 2019, while table $2 \mathrm{~b}$ shows the average annual GDP rate of OECD countries. Table $3 \mathrm{a}$ in the Annexure shows unemployment rate of 34 OECD countries, while table $3 \mathrm{~b}$ shows the average annual unemployment rate of OECD countries.

\section{Results}


The results of the correlation analysis are shown in Table 4 and 5 of the Annexure. From the data analysis, it is found that there is no correlation between government taxation, revenues, and employment in OECD countries. The data analysis shows that government taxation, revenues and employment in OECD countries is not related.

A change in the taxation was found to have no significant effect on the government revenues or employment in OECD countries during the 20 year period between 2000 and 2019.

\section{Conclusion \& Recommendation}

The data analysis shows that corporate taxation, government revenues, and employment in OECD countries are not related. No significant relationship was found between the study variables during the period 2000 and 2019. The results show that there might be other variables that explain the changes in taxation, government revenues and employment in OECD countries.

The results of the literature review showed that a decrease in taxation leads to improved investments. But most studies did not take account of aggregate taxes during a particular period. The findings of the study meet the aggregate tax elasticity model suggested by Deverux (2007a) and Brill and Hassett (2007) that a Laffer curve is attained when the corporate income tax hovers around 30 percent. Table $1 \mathrm{~b}$ shows that the average aggregate tax for the OECD countries during the period 2000 and 2019 is about 26.5 percent, which near the 30 percent. At the Laffer curve, the government income tax revenues would not be responsive to a change in the tax rate. The conclusion is not made by most studies reviewed since they have not assessed aggregate taxation rates.

The firm's investment choices during a particular period may be influenced by many factors. Further research is recommended to discover the latent, mediating, and moderating variables that affect the relationship between corporation taxation, government revenues and employment in OECD countries. The ex-ante tax reforms should be considered when assessing the impact of taxation on government revenues and employment levels within a country.

Hardenberg's uncertainty principle seems to apply in measuring the effect of the taxation. Corporate taxation is a complex topic and simplified empirical formulation cannot help in accurately recording the accurate position. Firms may have been given tax incentives or there might be tax agreements regarding tax payments in a particular location. There might also be taxation on profits of multinational firms that needs to be considered. Accounting for all the different factors is a complex endeavour that is beyond the scope and budget of this study.

Policy makers need to consider the fact that a significantly lower than average rate beyond the Laffer curve can result in increased investment. But the other relevant factors should be considered. For instance, the government must create a policy that encourage firm to invest in capital projects that will generate more revenues and investment rather than securities and bonds. A country can also benefit by cooperating with other countries regarding taxation of multinational firms.

The effect of the change in tax structure depends on the reaction of the firms. The study findings show that the aggregate taxation rate in OECD countries does not affect government revenues and employment. From the findings of the study, it can be concluded that various exogenous factors affect the relationship between corporate taxation, government revenues and employment levels within a country. All these factors need to be studied to create an effective fiscal policy to stimulate the economy.

Various measures can help ensure optimal corporate taxation policy. One effective measure to ensure optimal corporate tax rate is to create a plan that takes into account the potential effects of changes in the corporate taxation policy. The plan should be created with input from different departments in the public and private firms. Feedback should be taken from managers, business owners, and C-level executives regarding effects of a change in the taxation rate. The interdepartmental effort can help in creating an effective taxation policy that is based on specific benefits and risks identified by the professionals of different corporations. The objective of the taxation policy should be carefully monitored and changes should be made as necessary.

Implementing changes in the corporate policy is one of the challenging parts of corporate taxation policy. There might be negative impact of a possible increase in the corporate taxation that can have an adverse effect on the government revenues. Firms could take steps such as hiding revenue stream through creative accounting tactics to reduce the taxation. The actions of the corporations should be closely monitored to ensure that firms present an accurate picture about the financial position.

Creating a plan for effective corporate taxation for the initiative is just the first step of ensuring maximum benefits on the government revenues, corporate investment, and employment levels. A taxation plan should structure the funding sources for meeting the goals of the corporate taxation. The plan should drive the decision making and direction of the government's initiative in meeting the plan. This plan should identify short, medium, and long-run goals for changes in the corporate taxation. A plan should also include benchmarks to evaluate the effectiveness of the corporate policy changes. It should identify the factors that can have an effect on the implementation of any policy changes regarding corporate taxation. Any ramifications that may arise due to changes in the corporate policy should also be documented in the plan. Some of the potential challenges in the implementing plan include negative reaction from the corporate sector particularly multinational companies. These challenges can be overcome through a collaborative goal setting, proper communication, and continuous monitoring of the efforts to implement the plan. The taxation planning is important to ensure the best outcome from a change in the rates for boosting government revenues. 
It is important to overcome the challenges and objections to the implementation of the effective corporate taxation policy that have a positive impact on the government taxation revenues.

Disclosure of interest: The author reports no potential conflict of interest that could create bias or influence the outcome of the results.

\section{References}

Agarwal, S. \& Chakraborty, L. (2019). Business Taxation in an Emerging Economy: Analysing Corporate Tax Incidence. Review of European Studies, Canadian Center of Science and Education, 11(2), 1-8. http://www.ccsenet.org/journal/index.php/res/article/download/0/0/38909/40615

André, C. \&Hwang, H. (2018). Tax reform to support growth and employment in Finland. OECD Economics Department Working Papers 1468, OECD Publishing.https://doi.org/10.1787/412834d3-en

Arnold, J. (2008) Do tax structures affect aggregate economic growth? Empirical evidence from a panel of OECD countries. OECD Working Paper, 643. http://www. oecd- ilibrary.org/economics/do-tax-structures-affect-aggregateeconomic-growth_236001 777843 ? crawler =true.

Bajrami, E.(2017). The impact of corporate income tax on wages and employment. Academic Journal of Economic Studies, 3(2), 104-109. http://www.ajes.ro/wp-content/uploads/AJES_article_1_105.pdf

Bartik, T.J. (1985). Business Location Decisions in the United States: Estimates of the Effects ofUnionization, Taxes, and Other Characteristics of States.Journal of Business and Economic Statistics, 3, 14 22.https://www.tandfonline.com/doi/abs/10.1080/07350015.1985.10509422

Bernal, R., Eslava, M., Melendez, M., \& Pinzon, A. (2017). Switching from payroll taxes to corporate income taxes: Firms employment and wages after the 2012 Colombian tax reform. Economía Journal, 41-74. https://www.jstor.org/stable/90017435

Bodgan, D. \& Maria, D.S. (2017). Does Corporate Tax Burden Affect Growth? Evidences from OECD Countries.Journal of Heterodox Economics, Sciendo, 4(2), 51-80.https://doi.org/10.1515/jheec-2017-0004

Brill, A.\& Hassett, K.A. (2007). Revenue-Maximizing Corporate Income Taxes: The Laffer Curve in OECD Countries. Working Paper No. 137, American Enterprise Institute for Public Policy, Washington D.C.

Bua, M.,Girard, P., Legendre, F., \& Redondo, P. (1990). Les effets favorables d'une baisse de la fiscalité des entreprises : une évaluation à partir de données individuelles.Économie et Statistique, Programme National Persée, 229(1), 41-51.https://doi.org/10.3406/estat.1990.5419

Cai, K., Chen, Y. \& Wang, X. (2018). The impact of corporate taxes on firm innovation: Evidence from the corporate tax collection reform in China. https://voxeu.org/article/impact-corporate-taxes-firm-innovation

Chen, D., Qi, S., \& Schalgenhaulf, D.E. (2018). Corporate income tax, legal form of organization, and employment. American Economic Journal: Macroeconomics, 10(4), 270-304. https://doi.org/10.1257/mac.20140103

Cobham, A. \& Jansky, P. (2018). Global distribution of revenue loss from corporate tax avoidance: Reâlestimation and country results. Journal of International Development, 30(2), 206-232.https://doi.org/10.1002/jid.3348

Connolly, M. \& Li, Cheng. (2016). Government spending and economic growth in theOECD countries. Journal of Economic Policy Reform. https://doi.org/10.1080/17487870.2016.1213168

Devereux, M. P. (2007). Developments in the Taxation of Corporate Profit in the OECD since 1965: Rates, Bases and Revenues. Working Paper No. 0704, Oxford University Centre for Business Taxation.

Dwenger, N., Rattenhuber, P., \& Steiner, V. (2011). Sharing the burden: Empirical evidence on corporate tax incidence. Discussion Papers 2011/19, Free University Berlin, School of Business \& Economics. https://www.econstor.eu/bitstream/10419/54727/1/68054836X.pdf

Dwenger, N., Rattnehuber, P., \& Steiner, V. (2019). Sharing the burden? Empirical evidence on corporate tax incidence. German Economic Review, 20(4), 107-140. https://doi.org/10.1111/geer.12157

Fuest, C., Peichl, A., \& Siegloch, S. (2018). Do higher corporate taxes reduce wages? Micro evidence from Germany. American Economic, 108(2), 393-418. https://doi.org/10.1257/aer.20130570

Gober J.R., \& Burns J.O., (1997). The relationship between tax structures and economic indicators. Journal of International Accounting, Auditing \& Taxation, 6, 1-24. https://doi.org/10.1016/S1061-9518(97)90010-0

Harberger, Arnold C. (1962). The Incidence of the Corporation Income Tax.Journal of Political Economy, 70(3): 215-40.http://citeseerx.ist.psu.edu/viewdoc/summary?doi=10.1.1.198.4639

Harden, J. William \& Hoyt, William H. (2003). Do States Choose Their Mix of Taxes to Minimize Employment Losses? National Tax Journal, 56(1), 7-26.

Hassett, K.A., \& Mathur, A. (2015). A spatial model of corporate tax incidence. Applied Economics, 47(13), 13501365. https://doi.org/10.1080/00036846.2014.995367

Hines, J.R. (1996). Altered States: Taxes and the Location of Foreign Direct Investment in America. American Economic Review,86, 1076 - 1094.https://ideas.repec.org/a/aea/aecrev/v86y1996i5p1076-94.html

Hodge, S.A. (2016). The corporate income tax is most harmful for growth and wages. https://taxfoundation.org/corporate-income-tax-most-harmful-growth-and-wages/ 
International Monetary Fund (2002). Slovak Republic; Staff Report for the 2002 Article IV Consultation. IMF Staff Country Reports 2002/209. http://www.imf.org/external/pubs/cat/longres.aspx?sk=16084

Kapko, E. (2018). The corporate tax, appointment rules, and employment: Evidence using policy discontinuity at US state borders. MPRA Paper 94875, University Library of Munich, Germany.https://mpra.ub.unimuenchen.de/94875/1/MPRA_paper_94875.pdf

Kelly, J. \& Graziani, R. (2004). International trends in company tax rates - Implications for Australia's company income tax. Economic Roundup, 3, 23-47. http://archive.treasury.gov.au/documents/930/PDF/02_International.pdf

Koroviy, V. (2020). Tax regulation of socio-economic development. University Economic Bulletin, 47, 147-155. https://doi.org/10.31470/2306-546X-2020-47-147-155

Kouparitsas, M., Pirhardini, D., Beames, A. (2016). Analysis of the long term effects of a company tax cut. Treasury Working Papers 2016-02, The Treasury, Australian Government. http://www.treasury.gov.au/ /media/Treasury/Publications\%20and\%20Media/Publications/2016/TWP2/Downloads/PD F/Treasury-Working-Paper-2016-02.ashx

Leigh, A. (2018). Do firms that pay less company tax create more job? Economic Analysis Policy, 59(C), 25-28. https://doi.org/10.1016/j.eap.2018.02.003

Li, Bing, Liu, Chang \& Sun, Stephen Teng (2021). Do corporate income tax cuts decrease labor share? Regression discontinuity evidence from China. Journal of Development Economics,150(C). https://doi.org/ 10.1016/j.jdeveco.2021.102624

Lukes, J. \& Skalova, J. (2021). Misuse of company mergers in investment funds?. In David Procházka (ed.), Digitalization in Finance and Accounting, 181-188, Springer. https://doi.org/10.1007/978-3-030-55277-0_16

Mittermaier, F., \& Rincke, J. (2013). Do countries compensate firms for international wage differentials? Journal of Public Economics, 102, 23-36. https://doi.org/10.1016/j.jpubeco.2013.03.003

Mitroi, M. (2018). Repercursions of the implementation of fiscal policy's objectives on economic growth. Proceedings of the International Management Conference, Bucharest, Romania. Academy of Economic Studies 12(1), 495-504. http://conference.management.ase.ro/archives/2018/pdf/3_12.pdf

Nam, Chang \&Radulescu, Doina (2004). Do corporate tax concessions really matter for the success of free economic zones? Economic Change and Restructuring, 37(2), 99-123.https://link.springer.com/article/10.1007/s10644004-6831-6

Ohno, T.,Hotei, M., Sato, E., \& Umezaki, C. (2015). Decomposition of the variation of corporate tax revenues: Based on consideration of corporate tax paradox. Public Policy Review, Policy Research Institute, Ministry of Finance Japan, $\quad 11(2), \quad 333-360$. https://warp.da.ndl.go.jp/info:ndljp/pid/11217434/www.mof.go.jp/english/pri/publication/pp_review/ppr028/ppr028e.pd $\mathrm{f}$

Ohrn, E. (2018). The effect of corporate taxation on investment and financial policy. American Economic Journal: Economic Policy, 10(2), 272-301. https://www.jstor.org/stable/26529031?seq=1

Organization for Economic Cooperation and Development (n.d.). Tax and economic growth. Retrieved from https://www.oecd.org/mena/competitiveness/41997578.pdf

Papke, L.E. (1991), Interstate business tax differentials and new firm location: Evidence from panel data. Journal of Public Economics, 45, 47 - 68.https://econpapers.repec.org/article/eeepubeco/v_3a45_3ay_3a1991_3ai_3a1_3ap_3a4768.htm

Peichl, Andreas, Fuest, Clemens, \& Siegloch, Sebastian (2013). Wage Incidence of Local Corporate Taxation Micro Evidence from Germany. VfS Annual Conference 2013 (Duesseldorf): Competition Policy and Regulation in a Global Economic Order 79916. Verein für Socialpolitik / German Economic Association. https://www.econstor.eu/bitstream/10419/79916/1/VfS_2013_pid_590.pdf

Persson, T. \& Tabellini, G. (1994). Is inequality harmful for growth? American Economic Review, 84(3), 600621.https://www.jstor.org/stable/2118070

Preiss, Kenneth J. \&Spooner, K. (2003). Innovation creation and diffusion in the Australian economy. International Journal of Entrepreneurship and Innovation Management, 3(3), 197-210.http://www.inderscience.com/link.php?id=3286

Riedel, N. (2011) Taxing multinationals under union wage bargaining. Working Papers 1106, Oxford University Centre for Business Taxation. http://www.sbs.ox.ac.uk/sites/default/files/Business_Taxation/Docs/Publications/Working_Papers/Series_11/WP1106.p df

Strulik, H. \& Siegfried, N.A.(1999). A Financial theory of firm growth. Quantitative Macroeconomics Working Papers 19910, Hamburg University, Department of Economics.http://www.rrz.uni-hamburg.de/wst/qmwps/qm699.ps

Sen, Huseyin, \& Cevik, Z.B.C. (2021) The revenue-maximizing corporate income tax rate for Turkey. Journal for Economic Forecasting, Institute for Economic Forecasting, 0(1),122-142. http://www.ipe.ro/rjef/rjef1_21/rjef1_2021p122-142.pdf

Shakibaei, A. \& Ahmadinejad, M.R. (2016). Investigating the structural changes of tax in Iran. Iranian Economic Review (IER). 20(4), 445-460. https://ier.ut.ac.ir/article_59604_2d6cc4979143d32e23719f799c9c0af7.pdf 
Swank, D., \& Steinmo, S. (2002). The new political economy of taxation in advanced capitalist democracies. American Journal of Political Science, 46(3), 642-655. https://doi.org/10.2307/3088405

Uhlig, H., \& Yanagawa, N (1996) Increasing the capital income tax may lead to faster growth. European Economic Review, 40,1521-1540.http://home.uchicago.edu/ huhlig/papers/uhlig.yanagawa.eer.1996.pdf

Zeli, A. (2020). The evaluation of job tax incentives: An analysis of a regional tax. Canadian Tax Journal, 68(1), 131. https://doi.org/10.32721/ctj.2020.68.1.zeli 


\section{Annexure}

Table 1

Corporate Income Tax Rates of 34 OECD Countries, while table $2 \mathrm{~b}$ shows the average annual income tax rate of OECD countries.

\begin{tabular}{|c|c|c|c|c|c|c|c|c|c|c|c|c|c|}
\hline & 2000 & 2001 & 2002 & 2003 & 2004 & 2005 & 2006 & 2007 & 2008 & 2009 & 2010 & 2011 & 2012 \\
\hline Australia & $\begin{array}{c}34.0 \\
\%\end{array}$ & $\begin{array}{c}30.0 \\
\%\end{array}$ & $\begin{array}{c}30.0 \\
\%\end{array}$ & $\begin{array}{c}30.0 \\
\%\end{array}$ & $\begin{array}{c}30.0 \\
\%\end{array}$ & $\begin{array}{c}30.0 \\
\%\end{array}$ & $\begin{array}{c}30.0 \\
\%\end{array}$ & $\begin{array}{c}30.0 \\
\%\end{array}$ & $\begin{array}{c}30.0 \\
\%\end{array}$ & $\begin{array}{c}30.0 \\
\%\end{array}$ & $\begin{array}{c}30.0 \\
\%\end{array}$ & $\begin{array}{c}30.0 \\
\%\end{array}$ & $\begin{array}{c}30.0 \\
\%\end{array}$ \\
\hline Austria & $\begin{array}{c}34.0 \\
\%\end{array}$ & $\begin{array}{c}34.0 \\
\%\end{array}$ & $\begin{array}{c}34.0 \\
\%\end{array}$ & $\begin{array}{c}34.0 \\
\%\end{array}$ & $\begin{array}{c}34.0 \\
\%\end{array}$ & $\begin{array}{c}25.0 \\
\%\end{array}$ & $\begin{array}{c}25.0 \\
\%\end{array}$ & $\begin{array}{c}25.0 \\
\%\end{array}$ & $\begin{array}{c}25.0 \\
\%\end{array}$ & $\begin{array}{c}25.0 \\
\%\end{array}$ & $\begin{array}{c}25.0 \\
\%\end{array}$ & $\begin{array}{c}25.0 \\
\%\end{array}$ & $\begin{array}{c}25.0 \\
\%\end{array}$ \\
\hline Belgium & $\begin{array}{c}40.2 \\
\%\end{array}$ & $\begin{array}{c}40.2 \\
\%\end{array}$ & $\begin{array}{c}40.2 \\
\%\end{array}$ & $\begin{array}{c}34.0 \\
\%\end{array}$ & $\begin{array}{c}34.0 \\
\%\end{array}$ & $\begin{array}{c}34.0 \\
\%\end{array}$ & $\begin{array}{c}36.0 \\
\%\end{array}$ & $\begin{array}{c}34.0 \\
\%\end{array}$ & $\begin{array}{c}34.0 \\
\%\end{array}$ & $\begin{array}{c}34.0 \\
\%\end{array}$ & $\begin{array}{c}34.0 \\
\%\end{array}$ & $\begin{array}{c}34.0 \\
\%\end{array}$ & $\begin{array}{c}34.0 \\
\%\end{array}$ \\
\hline Canada & $\begin{array}{c}42.4 \\
\%\end{array}$ & $\begin{array}{c}40.5 \\
\%\end{array}$ & $\begin{array}{c}38.0 \\
\%\end{array}$ & $\begin{array}{c}35.9 \\
\%\end{array}$ & $\begin{array}{c}34.4 \\
\%\end{array}$ & $\begin{array}{c}34.2 \\
\%\end{array}$ & $\begin{array}{c}33.9 \\
\%\end{array}$ & $\begin{array}{c}34.0 \\
\%\end{array}$ & $\begin{array}{c}31.4 \\
\%\end{array}$ & $\begin{array}{c}30.9 \\
\%\end{array}$ & $\begin{array}{c}29.4 \\
\%\end{array}$ & $\begin{array}{c}27.7 \\
\%\end{array}$ & $\begin{array}{c}26.1 \\
\%\end{array}$ \\
\hline Chile & $\begin{array}{c}15.0 \\
\%\end{array}$ & $\begin{array}{c}15.0 \\
\%\end{array}$ & $\begin{array}{c}16.0 \\
\%\end{array}$ & $\begin{array}{c}16.5 \\
\%\end{array}$ & $\begin{array}{c}17.0 \\
\%\end{array}$ & $\begin{array}{c}17.0 \\
\%\end{array}$ & $\begin{array}{c}17.0 \\
\%\end{array}$ & $\begin{array}{c}17.0 \\
\%\end{array}$ & $\begin{array}{c}17.0 \\
\%\end{array}$ & $\begin{array}{c}17.0 \\
\%\end{array}$ & $\begin{array}{c}17.0 \\
\%\end{array}$ & $\begin{array}{c}20.0 \\
\%\end{array}$ & $\begin{array}{c}20.0 \\
\%\end{array}$ \\
\hline $\begin{array}{c}\text { Czech } \\
\text { Republic }\end{array}$ & $\begin{array}{c}31.0 \\
\%\end{array}$ & $\begin{array}{c}31.0 \\
\%\end{array}$ & $\begin{array}{c}31.0 \\
\%\end{array}$ & $\begin{array}{c}31.0 \\
\%\end{array}$ & $\begin{array}{c}28.0 \\
\%\end{array}$ & $\begin{array}{c}26.0 \\
\%\end{array}$ & $\begin{array}{c}24.0 \\
\%\end{array}$ & $\begin{array}{c}24.0 \\
\%\end{array}$ & $\begin{array}{c}21.0 \\
\%\end{array}$ & $\begin{array}{c}20.0 \\
\%\end{array}$ & $\begin{array}{c}19.0 \\
\%\end{array}$ & $\begin{array}{c}19.0 \\
\%\end{array}$ & $\begin{array}{c}19.0 \\
\%\end{array}$ \\
\hline Denmark & $\begin{array}{c}32.0 \\
\%\end{array}$ & $\begin{array}{c}30.0 \\
\%\end{array}$ & $\begin{array}{c}30.0 \\
\%\end{array}$ & $\begin{array}{c}30.0 \\
\%\end{array}$ & $\begin{array}{c}30.0 \\
\%\end{array}$ & $\begin{array}{c}28.0 \\
\%\end{array}$ & $\begin{array}{c}28.0 \\
\%\end{array}$ & $\begin{array}{c}25.0 \\
\%\end{array}$ & $\begin{array}{c}25.0 \\
\%\end{array}$ & $\begin{array}{c}25.0 \\
\%\end{array}$ & $\begin{array}{c}25.0 \\
\%\end{array}$ & $\begin{array}{c}25.0 \\
\%\end{array}$ & $\begin{array}{c}25.0 \\
\%\end{array}$ \\
\hline Estonia & $\begin{array}{c}26.0 \\
\%\end{array}$ & $\begin{array}{c}26.0 \\
\%\end{array}$ & $\begin{array}{c}26.0 \\
\%\end{array}$ & $\begin{array}{c}26.0 \\
\%\end{array}$ & $\begin{array}{c}26.0 \\
\%\end{array}$ & $\begin{array}{c}24.0 \\
\%\end{array}$ & $\begin{array}{c}23.0 \\
\%\end{array}$ & $\begin{array}{c}22.0 \\
\%\end{array}$ & $\begin{array}{c}21.0 \\
\%\end{array}$ & $\begin{array}{c}21.0 \\
\%\end{array}$ & $\begin{array}{c}21.0 \\
\%\end{array}$ & $\begin{array}{c}21.0 \\
\%\end{array}$ & $\begin{array}{c}21.0 \\
\%\end{array}$ \\
\hline Finland & $\begin{array}{c}29.0 \\
\%\end{array}$ & $\begin{array}{c}29.0 \\
\%\end{array}$ & $\begin{array}{c}29.0 \\
\%\end{array}$ & $\begin{array}{c}29.0 \\
\%\end{array}$ & $\begin{array}{c}29.0 \\
\%\end{array}$ & $\begin{array}{c}26.0 \\
\%\end{array}$ & $\begin{array}{c}26.0 \\
\%\end{array}$ & $\begin{array}{c}26.0 \\
\%\end{array}$ & $\begin{array}{c}26.0 \\
\%\end{array}$ & $\begin{array}{c}26.0 \\
\%\end{array}$ & $\begin{array}{c}26.0 \\
\%\end{array}$ & $\begin{array}{c}26.0 \\
\%\end{array}$ & $\begin{array}{c}24.5 \\
\%\end{array}$ \\
\hline France & $\begin{array}{c}37.8 \\
\%\end{array}$ & $\begin{array}{c}36.4 \\
\%\end{array}$ & $\begin{array}{c}35.4 \\
\%\end{array}$ & $\begin{array}{c}35.4 \\
\%\end{array}$ & $\begin{array}{c}35.4 \\
\%\end{array}$ & $\begin{array}{c}35.0 \\
\%\end{array}$ & $\begin{array}{c}34.4 \\
\%\end{array}$ & $\begin{array}{c}34.4 \\
\%\end{array}$ & $\begin{array}{c}34.4 \\
\%\end{array}$ & $\begin{array}{c}34.4 \\
\%\end{array}$ & $\begin{array}{c}34.4 \\
\%\end{array}$ & $\begin{array}{c}36.1 \\
\%\end{array}$ & $\begin{array}{c}36.1 \\
\%\end{array}$ \\
\hline Germany & $\begin{array}{c}51.6 \\
\%\end{array}$ & $\begin{array}{c}38.3 \\
\%\end{array}$ & $\begin{array}{c}38.3 \\
\%\end{array}$ & $\begin{array}{c}39.6 \\
\%\end{array}$ & $\begin{array}{c}38.3 \\
\%\end{array}$ & $\begin{array}{c}38.4 \\
\%\end{array}$ & $\begin{array}{c}38.4 \\
\%\end{array}$ & $\begin{array}{c}38.4 \\
\%\end{array}$ & $\begin{array}{c}29.4 \\
\%\end{array}$ & $\begin{array}{c}29.4 \\
\%\end{array}$ & $\begin{array}{c}29.5 \\
\%\end{array}$ & $\begin{array}{c}29.6 \\
\%\end{array}$ & $\begin{array}{c}29.6 \\
\%\end{array}$ \\
\hline Greece & $\begin{array}{c}40.0 \\
\%\end{array}$ & $\begin{array}{c}37.5 \\
\%\end{array}$ & $\begin{array}{c}35.0 \\
\%\end{array}$ & $\begin{array}{c}35.0 \\
\%\end{array}$ & $\begin{array}{c}35.0 \\
\%\end{array}$ & $\begin{array}{c}32.0 \\
\%\end{array}$ & $\begin{array}{c}29.0 \\
\%\end{array}$ & $\begin{array}{c}25.0 \\
\%\end{array}$ & $\begin{array}{c}25.0 \\
\%\end{array}$ & $\begin{array}{c}25.0 \\
\%\end{array}$ & $\begin{array}{c}24.0 \\
\%\end{array}$ & $\begin{array}{c}20.0 \\
\%\end{array}$ & $\begin{array}{c}20.0 \\
\%\end{array}$ \\
\hline Hungary & $\begin{array}{c}18.0 \\
\%\end{array}$ & $\begin{array}{c}18.0 \\
\%\end{array}$ & $\begin{array}{c}18.0 \\
\%\end{array}$ & $\begin{array}{c}18.0 \\
\%\end{array}$ & $\begin{array}{c}16.0 \\
\%\end{array}$ & $\begin{array}{c}16.0 \\
\%\end{array}$ & $\begin{array}{c}17.3 \\
\%\end{array}$ & $\begin{array}{c}20.0 \\
\%\end{array}$ & $\begin{array}{c}20.0 \\
\%\end{array}$ & $\begin{array}{c}20.0 \\
\%\end{array}$ & $\begin{array}{c}19.0 \\
\%\end{array}$ & $\begin{array}{c}19.0 \\
\%\end{array}$ & $\begin{array}{c}19.0 \\
\%\end{array}$ \\
\hline Iceland & $\begin{array}{c}30.0 \\
\%\end{array}$ & $\begin{array}{c}30.0 \\
\%\end{array}$ & $\begin{array}{c}18.0 \\
\%\end{array}$ & $\begin{array}{c}18.0 \\
\%\end{array}$ & $\begin{array}{c}18.0 \\
\%\end{array}$ & $\begin{array}{c}18.0 \\
\%\end{array}$ & $\begin{array}{c}18.0 \\
\%\end{array}$ & $\begin{array}{c}18.0 \\
\%\end{array}$ & $\begin{array}{c}15.0 \\
\%\end{array}$ & $\begin{array}{c}15.0 \\
\%\end{array}$ & $\begin{array}{c}18.0 \\
\%\end{array}$ & $\begin{array}{c}20.0 \\
\%\end{array}$ & $\begin{array}{c}20.0 \\
\%\end{array}$ \\
\hline Ireland & $\begin{array}{c}24.0 \\
\%\end{array}$ & $\begin{array}{c}20.0 \\
\%\end{array}$ & $\begin{array}{c}16.0 \\
\%\end{array}$ & $\begin{array}{c}12.5 \\
\%\end{array}$ & $\begin{array}{c}12.5 \\
\%\end{array}$ & $\begin{array}{c}12.5 \\
\%\end{array}$ & $\begin{array}{c}12.5 \\
\%\end{array}$ & $\begin{array}{c}12.5 \\
\%\end{array}$ & $\begin{array}{c}12.5 \\
\%\end{array}$ & $\begin{array}{c}12.5 \\
\%\end{array}$ & $\begin{array}{c}12.5 \\
\%\end{array}$ & $\begin{array}{c}12.5 \\
\%\end{array}$ & $\begin{array}{c}12.5 \\
\%\end{array}$ \\
\hline Israel & $\begin{array}{c}36.0 \\
\%\end{array}$ & $\begin{array}{c}36.0 \\
\%\end{array}$ & $\begin{array}{c}36.0 \\
\%\end{array}$ & $\begin{array}{c}36.0 \\
\%\end{array}$ & $\begin{array}{c}35.0 \\
\%\end{array}$ & $\begin{array}{c}34.0 \\
\%\end{array}$ & $\begin{array}{c}31.0 \\
\%\end{array}$ & $\begin{array}{c}29.0 \\
\%\end{array}$ & $\begin{array}{c}27.0 \\
\%\end{array}$ & $\begin{array}{c}26.0 \\
\%\end{array}$ & $\begin{array}{c}25.0 \\
\%\end{array}$ & $\begin{array}{c}24.0 \\
\%\end{array}$ & $\begin{array}{c}25.0 \\
\%\end{array}$ \\
\hline Italy & $\begin{array}{c}41.3 \\
\%\end{array}$ & $\begin{array}{c}40.3 \\
\%\end{array}$ & $\begin{array}{c}40.3 \\
\%\end{array}$ & $\begin{array}{c}38.3 \\
\%\end{array}$ & $\begin{array}{c}37.3 \\
\%\end{array}$ & $\begin{array}{c}37.3 \\
\%\end{array}$ & $\begin{array}{c}37.3 \\
\%\end{array}$ & $\begin{array}{c}37.3 \\
\%\end{array}$ & $\begin{array}{c}31.4 \\
\%\end{array}$ & $\begin{array}{c}31.4 \\
\%\end{array}$ & $\begin{array}{c}31.4 \\
\%\end{array}$ & $\begin{array}{c}31.4 \\
\%\end{array}$ & $\begin{array}{c}31.3 \\
\%\end{array}$ \\
\hline Japan & $\begin{array}{c}40.9 \\
\%\end{array}$ & $\begin{array}{c}40.9 \\
\%\end{array}$ & $\begin{array}{c}40.9 \\
\%\end{array}$ & $\begin{array}{c}40.9 \\
\%\end{array}$ & $\begin{array}{c}39.5 \\
\%\end{array}$ & $\begin{array}{c}39.5 \\
\%\end{array}$ & $\begin{array}{c}39.5 \\
\%\end{array}$ & $\begin{array}{c}39.5 \\
\%\end{array}$ & $\begin{array}{c}39.5 \\
\%\end{array}$ & $\begin{array}{c}39.5 \\
\%\end{array}$ & $\begin{array}{c}39.5 \\
\%\end{array}$ & $\begin{array}{c}39.5 \\
\%\end{array}$ & $\begin{array}{c}39.5 \\
\%\end{array}$ \\
\hline $\begin{array}{l}\text { South } \\
\text { Korea }\end{array}$ & $\begin{array}{c}30.8 \\
\%\end{array}$ & $\begin{array}{c}30.8 \\
\%\end{array}$ & $\begin{array}{c}29.7 \\
\%\end{array}$ & $\begin{array}{c}29.7 \\
\%\end{array}$ & $\begin{array}{c}29.7 \\
\%\end{array}$ & $\begin{array}{c}27.5 \\
\%\end{array}$ & $\begin{array}{c}27.5 \\
\%\end{array}$ & $\begin{array}{c}27.5 \\
\%\end{array}$ & $\begin{array}{c}27.5 \\
\%\end{array}$ & $\begin{array}{c}24.2 \\
\%\end{array}$ & $\begin{array}{c}24.2 \\
\%\end{array}$ & $\begin{array}{c}24.2 \\
\%\end{array}$ & $\begin{array}{c}24.2 \\
\%\end{array}$ \\
\hline Latvia & $\begin{array}{c}25.0 \\
\%\end{array}$ & $\begin{array}{c}25.0 \\
\%\end{array}$ & $\begin{array}{c}22.0 \\
\%\end{array}$ & $\begin{array}{c}19.0 \\
\%\end{array}$ & $\begin{array}{c}15.0 \\
\%\end{array}$ & $\begin{array}{c}15.0 \\
\%\end{array}$ & $\begin{array}{c}15.0 \\
\%\end{array}$ & $\begin{array}{c}15.0 \\
\%\end{array}$ & $\begin{array}{c}15.0 \\
\%\end{array}$ & $\begin{array}{c}15.0 \\
\%\end{array}$ & $\begin{array}{c}15.0 \\
\%\end{array}$ & $\begin{array}{c}15.0 \\
\%\end{array}$ & $\begin{array}{c}15.0 \\
\%\end{array}$ \\
\hline Lithuania & $\begin{array}{c}24.0 \\
\%\end{array}$ & $\begin{array}{c}24.0 \\
\%\end{array}$ & $\begin{array}{c}15.0 \\
\%\end{array}$ & $\begin{array}{c}15.0 \\
\%\end{array}$ & $\begin{array}{c}15.0 \\
\%\end{array}$ & $\begin{array}{c}15.0 \\
\%\end{array}$ & $\begin{array}{c}19.0 \\
\%\end{array}$ & $\begin{array}{c}18.0 \\
\%\end{array}$ & $\begin{array}{c}15.0 \\
\%\end{array}$ & $\begin{array}{c}20.0 \\
\%\end{array}$ & $\begin{array}{c}15.0 \\
\%\end{array}$ & $\begin{array}{c}15.0 \\
\%\end{array}$ & $\begin{array}{c}15.0 \\
\%\end{array}$ \\
\hline $\begin{array}{c}\text { Luxembour } \\
\mathrm{g}\end{array}$ & $\begin{array}{c}37.5 \\
\%\end{array}$ & $\begin{array}{c}37.5 \\
\%\end{array}$ & $\begin{array}{c}30.4 \\
\%\end{array}$ & $\begin{array}{c}30.4 \\
\%\end{array}$ & $\begin{array}{c}30.4 \\
\%\end{array}$ & $\begin{array}{c}30.4 \\
\%\end{array}$ & $\begin{array}{c}29.6 \\
\%\end{array}$ & $\begin{array}{c}29.6 \\
\%\end{array}$ & $\begin{array}{c}29.6 \\
\%\end{array}$ & $\begin{array}{c}28.6 \\
\%\end{array}$ & $\begin{array}{c}28.6 \\
\%\end{array}$ & $\begin{array}{c}28.8 \\
\%\end{array}$ & $\begin{array}{c}28.8 \\
\%\end{array}$ \\
\hline Mexico & $\begin{array}{c}35.0 \\
\%\end{array}$ & $\begin{array}{c}35.0 \\
\%\end{array}$ & $\begin{array}{c}35.0 \\
\%\end{array}$ & $\begin{array}{c}34.0 \\
\%\end{array}$ & $\begin{array}{c}33.0 \\
\%\end{array}$ & $\begin{array}{c}30.0 \\
\%\end{array}$ & $\begin{array}{c}29.0 \\
\%\end{array}$ & $\begin{array}{c}28.0 \\
\%\end{array}$ & $\begin{array}{c}28.0 \\
\%\end{array}$ & $\begin{array}{c}28.0 \\
\%\end{array}$ & $\begin{array}{c}30.0 \\
\%\end{array}$ & $\begin{array}{c}30.0 \\
\%\end{array}$ & $\begin{array}{c}30.0 \\
\%\end{array}$ \\
\hline Netherlands & $\begin{array}{c}35.0 \\
\%\end{array}$ & $\begin{array}{c}35.0 \\
\%\end{array}$ & $\begin{array}{c}34.5 \\
\%\end{array}$ & $\begin{array}{c}34.5 \\
\%\end{array}$ & $\begin{array}{c}34.5 \\
\%\end{array}$ & $\begin{array}{c}31.5 \\
\%\end{array}$ & $\begin{array}{c}29.6 \\
\%\end{array}$ & $\begin{array}{c}25.5 \\
\%\end{array}$ & $\begin{array}{c}25.5 \\
\%\end{array}$ & $\begin{array}{c}25.5 \\
\%\end{array}$ & $\begin{array}{c}25.5 \\
\%\end{array}$ & $\begin{array}{c}25.0 \\
\%\end{array}$ & $\begin{array}{c}25.0 \\
\%\end{array}$ \\
\hline $\begin{array}{c}\text { New } \\
\text { Zealand }\end{array}$ & $\begin{array}{c}33.0 \\
\%\end{array}$ & $\begin{array}{c}33.0 \\
\%\end{array}$ & $\begin{array}{c}33.0 \\
\%\end{array}$ & $\begin{array}{c}33.0 \\
\%\end{array}$ & $\begin{array}{c}33.0 \\
\%\end{array}$ & $\begin{array}{c}33.0 \\
\%\end{array}$ & $\begin{array}{c}33.0 \\
\%\end{array}$ & $\begin{array}{c}33.0 \\
\%\end{array}$ & $\begin{array}{c}30.0 \\
\%\end{array}$ & $\begin{array}{c}30.0 \\
\%\end{array}$ & $\begin{array}{c}30.0 \\
\%\end{array}$ & $\begin{array}{c}28.0 \\
\%\end{array}$ & $\begin{array}{c}28.0 \\
\%\end{array}$ \\
\hline
\end{tabular}




\begin{tabular}{|c|c|c|c|c|c|c|c|c|c|c|c|c|c|}
\hline Norway & $\begin{array}{c}28.0 \\
\%\end{array}$ & $\begin{array}{c}28.0 \\
\%\end{array}$ & $\begin{array}{c}28.0 \\
\%\end{array}$ & $\begin{array}{c}28.0 \\
\%\end{array}$ & $\begin{array}{c}28.0 \\
\%\end{array}$ & $\begin{array}{c}28.0 \\
\%\end{array}$ & $\begin{array}{c}28.0 \\
\%\end{array}$ & $\begin{array}{c}28.0 \\
\%\end{array}$ & $\begin{array}{c}28.0 \\
\%\end{array}$ & $\begin{array}{c}28.0 \\
\%\end{array}$ & $\begin{array}{c}28.0 \\
\%\end{array}$ & $\begin{array}{c}28.0 \\
\%\end{array}$ & $\begin{array}{c}28.0 \\
\%\end{array}$ \\
\hline Poland & $\begin{array}{c}30.0 \\
\%\end{array}$ & $\begin{array}{c}28.0 \\
\%\end{array}$ & $\begin{array}{c}28.0 \\
\%\end{array}$ & $\begin{array}{c}27.0 \\
\%\end{array}$ & $\begin{array}{c}19.0 \\
\%\end{array}$ & $\begin{array}{c}19.0 \\
\%\end{array}$ & $\begin{array}{c}19.0 \\
\%\end{array}$ & $\begin{array}{c}19.0 \\
\%\end{array}$ & $\begin{array}{c}19.0 \\
\%\end{array}$ & $\begin{array}{c}19.0 \\
\%\end{array}$ & $\begin{array}{c}19.0 \\
\%\end{array}$ & $\begin{array}{c}19.0 \\
\%\end{array}$ & $\begin{array}{c}19.0 \\
\%\end{array}$ \\
\hline Portugal & $\begin{array}{c}35.2 \\
\%\end{array}$ & $\begin{array}{c}35.2 \\
\%\end{array}$ & $\begin{array}{c}33.0 \\
\%\end{array}$ & $\begin{array}{c}33.0 \\
\%\end{array}$ & $\begin{array}{c}27.5 \\
\%\end{array}$ & $\begin{array}{c}27.5 \\
\%\end{array}$ & $\begin{array}{c}27.5 \\
\%\end{array}$ & $\begin{array}{c}26.5 \\
\%\end{array}$ & $\begin{array}{c}26.5 \\
\%\end{array}$ & $\begin{array}{c}26.5 \\
\%\end{array}$ & $\begin{array}{c}26.5 \\
\%\end{array}$ & $\begin{array}{c}28.5 \\
\%\end{array}$ & $\begin{array}{c}31.5 \\
\%\end{array}$ \\
\hline $\begin{array}{c}\text { Slovak } \\
\text { Republic }\end{array}$ & $\begin{array}{c}29.0 \\
\%\end{array}$ & $\begin{array}{c}29.0 \\
\%\end{array}$ & $\begin{array}{c}25.0 \\
\%\end{array}$ & $\begin{array}{c}25.0 \\
\%\end{array}$ & $\begin{array}{c}19.0 \\
\%\end{array}$ & $\begin{array}{c}19.0 \\
\%\end{array}$ & $\begin{array}{c}19.0 \\
\%\end{array}$ & $\begin{array}{c}19.0 \\
\%\end{array}$ & $\begin{array}{c}19.0 \\
\%\end{array}$ & $\begin{array}{c}19.0 \\
\%\end{array}$ & $\begin{array}{c}19.0 \\
\%\end{array}$ & $\begin{array}{c}19.0 \\
\%\end{array}$ & $\begin{array}{c}19.0 \\
\%\end{array}$ \\
\hline Slovenia & $\begin{array}{c}25.0 \\
\%\end{array}$ & $\begin{array}{c}25.0 \\
\%\end{array}$ & $\begin{array}{c}25.0 \\
\%\end{array}$ & $\begin{array}{c}25.0 \\
\%\end{array}$ & $\begin{array}{c}25.0 \\
\%\end{array}$ & $\begin{array}{c}25.0 \\
\%\end{array}$ & $\begin{array}{c}25.0 \\
\%\end{array}$ & $\begin{array}{c}23.0 \\
\%\end{array}$ & $\begin{array}{c}22.0 \\
\%\end{array}$ & $\begin{array}{c}21.0 \\
\%\end{array}$ & $\begin{array}{c}20.0 \\
\%\end{array}$ & $\begin{array}{c}20.0 \\
\%\end{array}$ & $\begin{array}{c}18.0 \\
\%\end{array}$ \\
\hline Spain & $\begin{array}{c}35.0 \\
\%\end{array}$ & $\begin{array}{c}35.0 \\
\%\end{array}$ & $\begin{array}{c}35.0 \\
\%\end{array}$ & $\begin{array}{c}35.0 \\
\%\end{array}$ & $\begin{array}{c}35.0 \\
\%\end{array}$ & $\begin{array}{c}35.0 \\
\%\end{array}$ & $\begin{array}{c}35.0 \\
\%\end{array}$ & $\begin{array}{c}32.5 \\
\%\end{array}$ & $\begin{array}{c}30.0 \\
\%\end{array}$ & $\begin{array}{c}30.0 \\
\%\end{array}$ & $\begin{array}{c}30.0 \\
\%\end{array}$ & $\begin{array}{c}30.0 \\
\%\end{array}$ & $\begin{array}{c}30.0 \\
\%\end{array}$ \\
\hline Sweden & $\begin{array}{c}28.0 \\
\%\end{array}$ & $\begin{array}{c}28.0 \\
\%\end{array}$ & $\begin{array}{c}28.0 \\
\%\end{array}$ & $\begin{array}{c}28.0 \\
\%\end{array}$ & $\begin{array}{c}28.0 \\
\%\end{array}$ & $\begin{array}{c}28.0 \\
\%\end{array}$ & $\begin{array}{c}28.0 \\
\%\end{array}$ & $\begin{array}{c}28.0 \\
\%\end{array}$ & $\begin{array}{c}28.0 \\
\%\end{array}$ & $\begin{array}{c}26.3 \\
\%\end{array}$ & $\begin{array}{c}26.3 \\
\%\end{array}$ & $\begin{array}{c}26.3 \\
\%\end{array}$ & $\begin{array}{c}26.3 \\
\%\end{array}$ \\
\hline $\begin{array}{c}\text { United } \\
\text { Kingdom }\end{array}$ & $\begin{array}{c}30.0 \\
\%\end{array}$ & $\begin{array}{c}30.0 \\
\%\end{array}$ & $\begin{array}{c}30.0 \\
\%\end{array}$ & $\begin{array}{c}30.0 \\
\%\end{array}$ & $\begin{array}{c}30.0 \\
\%\end{array}$ & $\begin{array}{c}30.0 \\
\%\end{array}$ & $\begin{array}{c}30.0 \\
\%\end{array}$ & $\begin{array}{c}30.0 \\
\%\end{array}$ & $\begin{array}{c}28.0 \\
\%\end{array}$ & $\begin{array}{c}28.0 \\
\%\end{array}$ & $\begin{array}{c}28.0 \\
\%\end{array}$ & $\begin{array}{c}26.0 \\
\%\end{array}$ & $\begin{array}{c}24.0 \\
\%\end{array}$ \\
\hline $\begin{array}{l}\text { United } \\
\text { States }\end{array}$ & $\begin{array}{c}39.3 \\
\%\end{array}$ & $\begin{array}{c}39.3 \\
\%\end{array}$ & $\begin{array}{c}39.3 \\
\%\end{array}$ & $\begin{array}{c}39.3 \\
\%\end{array}$ & $\begin{array}{c}39.3 \\
\%\end{array}$ & $\begin{array}{c}39.3 \\
\%\end{array}$ & $\begin{array}{c}39.3 \\
\%\end{array}$ & $\begin{array}{c}39.3 \\
\%\end{array}$ & $\begin{array}{c}39.3 \\
\%\end{array}$ & $\begin{array}{c}39.2 \\
\%\end{array}$ & $\begin{array}{c}39.2 \\
\%\end{array}$ & $\begin{array}{c}39.2 \\
\%\end{array}$ & $\begin{array}{c}39.1 \\
\%\end{array}$ \\
\hline
\end{tabular}

\begin{tabular}{|c|c|c|c|c|c|c|c|}
\hline & 2013 & 2014 & 2015 & 2016 & 2017 & 2018 & 2019 \\
\hline & & & & & & & \\
\hline Australia & $30.0 \%$ & $30.0 \%$ & $30.0 \%$ & $30.0 \%$ & $30.0 \%$ & $30.0 \%$ & $30.0 \%$ \\
\hline Austria & $25.0 \%$ & $25.0 \%$ & $25.0 \%$ & $25.0 \%$ & $25.0 \%$ & $25.0 \%$ & $25.0 \%$ \\
\hline Belgium & $34.0 \%$ & $34.0 \%$ & $34.0 \%$ & $34.0 \%$ & $34.0 \%$ & $29.6 \%$ & $29.6 \%$ \\
\hline Canada & $26.2 \%$ & $26.2 \%$ & $26.7 \%$ & $26.7 \%$ & $26.7 \%$ & $26.8 \%$ & $26.6 \%$ \\
\hline Chile & $20.0 \%$ & $21.0 \%$ & $22.5 \%$ & $24.0 \%$ & $25.0 \%$ & $25.0 \%$ & $25.0 \%$ \\
\hline
\end{tabular}

\begin{tabular}{|c|c|c|c|c|c|c|c|}
\hline Czech Republic & $19.0 \%$ & $19.0 \%$ & $19.0 \%$ & $19.0 \%$ & $19.0 \%$ & $19.0 \%$ & $19.0 \%$ \\
\hline Denmark & $25.0 \%$ & $24.5 \%$ & $23.5 \%$ & $22.0 \%$ & $22.0 \%$ & $22.0 \%$ & $22.0 \%$ \\
\hline Estonia & $21.0 \%$ & $21.0 \%$ & $20.0 \%$ & $20.0 \%$ & $20.0 \%$ & $20.0 \%$ & $20.0 \%$ \\
\hline Finland & $24.5 \%$ & $20.0 \%$ & $20.0 \%$ & $20.0 \%$ & $20.0 \%$ & $20.0 \%$ & $20.0 \%$ \\
\hline France & $38.0 \%$ & $38.0 \%$ & $38.0 \%$ & $34.4 \%$ & $44.4 \%$ & $34.4 \%$ & $34.4 \%$ \\
\hline Germany & $29.6 \%$ & $29.7 \%$ & $29.8 \%$ & $29.8 \%$ & $29.9 \%$ & $29.9 \%$ & $29.9 \%$ \\
\hline Greece & $26.0 \%$ & $26.0 \%$ & $29.0 \%$ & $29.0 \%$ & $29.0 \%$ & $29.0 \%$ & $24.0 \%$ \\
\hline Hungary & $19.0 \%$ & $19.0 \%$ & $19.0 \%$ & $19.0 \%$ & $9.0 \%$ & $9.0 \%$ & $9.0 \%$ \\
\hline Iceland & $20.0 \%$ & $20.0 \%$ & $20.0 \%$ & $20.0 \%$ & $20.0 \%$ & $20.0 \%$ & $20.0 \%$ \\
\hline Ireland & $12.5 \%$ & $12.5 \%$ & $12.5 \%$ & $12.5 \%$ & $12.5 \%$ & $12.5 \%$ & $12.5 \%$ \\
\hline Israel & $25.0 \%$ & $26.5 \%$ & $26.5 \%$ & $25.0 \%$ & $24.0 \%$ & $23.0 \%$ & $23.0 \%$ \\
\hline Italy & $31.3 \%$ & $31.3 \%$ & $31.3 \%$ & $31.3 \%$ & $27.8 \%$ & $27.8 \%$ & $27.8 \%$ \\
\hline Japan & $37.0 \%$ & $37.0 \%$ & $32.1 \%$ & $30.0 \%$ & $30.0 \%$ & $29.7 \%$ & $29.7 \%$ \\
\hline South Korea & $24.2 \%$ & $24.2 \%$ & $24.2 \%$ & $24.2 \%$ & $24.2 \%$ & $27.5 \%$ & $27.5 \%$ \\
\hline Latvia & $15.0 \%$ & $15.0 \%$ & $15.0 \%$ & $15.0 \%$ & $15.0 \%$ & $20.0 \%$ & $20.0 \%$ \\
\hline
\end{tabular}




\begin{tabular}{|c|c|c|c|c|c|c|c|}
\hline Lithuania & $15.0 \%$ & $15.0 \%$ & $15.0 \%$ & $15.0 \%$ & $15.0 \%$ & $15.0 \%$ & $15.0 \%$ \\
\hline Luxembourg & $29.2 \%$ & $29.2 \%$ & $29.2 \%$ & $29.2 \%$ & $27.1 \%$ & $26.0 \%$ & $24.9 \%$ \\
\hline Mexico & $30.0 \%$ & $30.0 \%$ & $30.0 \%$ & $30.0 \%$ & $30.0 \%$ & $30.0 \%$ & $30.0 \%$ \\
\hline Netherlands & $25.0 \%$ & $25.0 \%$ & $25.0 \%$ & $25.0 \%$ & $25.0 \%$ & $25.0 \%$ & $25.0 \%$ \\
\hline New Zealand & $28.0 \%$ & $28.0 \%$ & $28.0 \%$ & $28.0 \%$ & $28.0 \%$ & $28.0 \%$ & $28.0 \%$ \\
\hline Norway & $28.0 \%$ & $27.0 \%$ & $27.0 \%$ & $25.0 \%$ & $24.0 \%$ & $23.0 \%$ & $22.0 \%$ \\
\hline Poland & $19.0 \%$ & $19.0 \%$ & $19.0 \%$ & $19.0 \%$ & $19.0 \%$ & $19.0 \%$ & $19.0 \%$ \\
\hline Portugal & $31.5 \%$ & $31.5 \%$ & $29.5 \%$ & $29.5 \%$ & $29.5 \%$ & $31.5 \%$ & $31.5 \%$ \\
\hline
\end{tabular}

\begin{tabular}{|c|l|l|l|l|l|l|l|}
\hline Slovak Republic & $23.0 \%$ & $22.0 \%$ & $22.0 \%$ & $22.0 \%$ & $21.0 \%$ & $21.0 \%$ & $21.0 \%$ \\
\hline Slovenia & $17.0 \%$ & $17.0 \%$ & $17.0 \%$ & $17.0 \%$ & $19.0 \%$ & $19.0 \%$ & $19.0 \%$ \\
\hline Spain & $30.0 \%$ & $30.0 \%$ & $28.0 \%$ & $25.0 \%$ & $25.0 \%$ & $25.0 \%$ & $25.0 \%$ \\
\hline Sweden & $22.0 \%$ & $22.0 \%$ & $22.0 \%$ & $22.0 \%$ & $22.0 \%$ & $22.0 \%$ & $21.4 \%$ \\
\hline United Kingdom & $23.0 \%$ & $21.0 \%$ & $20.0 \%$ & $20.0 \%$ & $19.0 \%$ & $19.0 \%$ & $19.0 \%$ \\
\hline United States & $39.0 \%$ & $39.1 \%$ & $39.0 \%$ & $38.9 \%$ & $38.9 \%$ & $25.8 \%$ & $25.9 \%$ \\
\hline
\end{tabular}

Source: OECD, Tax Database: https://stats.oecd.org/

Table $1 \mathrm{~b}$

Historical average Tax rate of 34 OECD Countries

\begin{tabular}{|c|c|c|c|c|c|c|c|c|c|c|c|c|c|}
\hline Year & 2000 & 2001 & 2002 & 2003 & 2004 & 2005 & 2006 & 2007 & 2008 & 2009 & 2010 & 2011 & 2012 \\
\hline $\begin{array}{c}\text { Incom } \\
\text { e Tax } \\
(\%)\end{array}$ & $\begin{array}{c}32.2 \\
\%\end{array}$ & $\begin{array}{c}31.3 \\
\%\end{array}$ & $\begin{array}{c}30.0 \\
\%\end{array}$ & $\begin{array}{c}29.4 \\
\%\end{array}$ & $\begin{array}{c}28.6 \\
\%\end{array}$ & $\begin{array}{c}27.5 \\
\%\end{array}$ & $\begin{array}{c}27.1 \\
\%\end{array}$ & $\begin{array}{c}26.5 \\
\%\end{array}$ & $\begin{array}{c}25.5 \\
\%\end{array}$ & $\begin{array}{c}25.3 \\
\%\end{array}$ & $\begin{array}{c}25.1 \\
\%\end{array}$ & $\begin{array}{c}25.1 \\
\%\end{array}$ & $\begin{array}{c}25.0 \\
\%\end{array}$ \\
\hline
\end{tabular}

\begin{tabular}{|c|c|c|c|c|c|c|c|}
\hline Year & 2013 & 2014 & 2015 & 2016 & 2017 & 2018 & 2019 \\
\hline Income Tax (\%) & $25.1 \%$ & $24.9 \%$ & $24.7 \%$ & $24.4 \%$ & $24.2 \%$ & $23.7 \%$ & $23.5 \%$ \\
\hline
\end{tabular}

Source: OECD, Tax Database: https://stats.oecd.org/

Table 2 a

Historical GDP of 34 OECD Countries

\begin{tabular}{|c|c|c|c|c|c|c|c|c|c|c|c|c|c|}
\hline & 2000 & 2001 & 2002 & 2003 & 2004 & 2005 & 2006 & 2007 & 2008 & 2009 & 2010 & 2011 & 2012 \\
\hline & & & & & & & & & & & & & \\
\hline Austr & 28252 & 29493 & 30740 & 32248 & 33865 & 35570 & 37842 & 39596 & 40094 & 41614 & 42787 & 44419 & 43879 \\
alia & .63 & .21 & .96 & .26 & .91 & .81 & .93 & .04 & .83 & .09 & .07 & .31 & .18 \\
\hline Austri & 29388 & 29715 & 31178 & 32123 & 33756 & 35024 & 37614 & 39384 & 41316 & 40957 & 42053 & 44469 & 46477 \\
a & .78 & .64 & .05 & .7 & .26 & .56 & .94 & .19 & .02 & .26 & .24 & .21 & .66 \\
\hline Belgi & 27797 & 28799 & 30281 & 30901 & 32036 & 33176 & 35211 & 36745 & 37883 & 37779 & 39868 & 40943 & 42290 \\
um & .33 & .33 & .67 & .38 & .94 & .68 & .89 & .5 & .23 & .03 & .99 & .34 & .48 \\
\hline Canad & 29363 & 30214 & 30963 & 32333 & 33911 & 36327 & 38107 & 39555 & 40376 & 38893 & 40114 & 41666 & 42290 \\
a & .15 & .3 & .22 & .61 & .22 & .66 & .14 & .52 & .33 & .73 & .22 & .72 & .88 \\
\hline Chile & 9519 & 9937. & 10292 & 10793 & 11757 & 12667 & 15685 & 16872 & 16447 & 16119 & 18129 & 20302 & 21446 \\
& 174 & 065 & .92 & .77 & .51 & .85 & .22 & .27 & .33 & .89 & .09 & .79 & .54 \\
\hline
\end{tabular}




\begin{tabular}{|c|c|c|c|c|c|c|c|c|c|c|c|c|c|}
\hline $\begin{array}{c}\text { Czech } \\
\text { Repub } \\
\text { lic }\end{array}$ & $\begin{array}{c}16214 \\
.97\end{array}$ & $\begin{array}{c}17615 \\
.6\end{array}$ & $\begin{array}{c}18245 \\
.67\end{array}$ & $\begin{array}{c}19503 \\
.29\end{array}$ & $\begin{array}{c}20894 \\
.61\end{array}$ & 22046 & $\begin{array}{c}23827 \\
.15\end{array}$ & $\begin{array}{c}26189 \\
.8\end{array}$ & $\begin{array}{c}27853 \\
.55\end{array}$ & $\begin{array}{c}27656 \\
.02\end{array}$ & $\begin{array}{c}27789 \\
.61\end{array}$ & $\begin{array}{c}28999 \\
.75\end{array}$ & $\begin{array}{c}29258 \\
.9\end{array}$ \\
\hline $\begin{array}{c}\text { Denm } \\
\text { ark }\end{array}$ & $\begin{array}{c}28679 \\
.27\end{array}$ & $\begin{array}{c}29459 \\
.85\end{array}$ & $\begin{array}{c}30639 \\
.95\end{array}$ & $\begin{array}{c}30791 \\
.9\end{array}$ & $\begin{array}{c}32920 \\
.68\end{array}$ & $\begin{array}{c}34152 \\
.88\end{array}$ & $\begin{array}{c}37286 \\
.32\end{array}$ & $\begin{array}{c}38969 \\
.12\end{array}$ & 41283 & $\begin{array}{c}40361 \\
.79\end{array}$ & $\begin{array}{c}43041 \\
.19\end{array}$ & $\begin{array}{c}44407 \\
.94\end{array}$ & $\begin{array}{c}44808 \\
.55\end{array}$ \\
\hline
\end{tabular}

\begin{tabular}{|c|c|c|c|c|c|c|c|c|c|c|c|c|c|}
\hline Estonia & $\begin{array}{c}9408 . \\
747\end{array}$ & $\begin{array}{c}10292 \\
.33\end{array}$ & $\begin{array}{c}11635 \\
.37\end{array}$ & $\begin{array}{c}13081 \\
.09\end{array}$ & $\begin{array}{c}14482 \\
.63\end{array}$ & $\begin{array}{c}16574 \\
.46\end{array}$ & $\begin{array}{c}19251 \\
.86\end{array}$ & $\begin{array}{c}22128 \\
.13\end{array}$ & $\begin{array}{c}22807 \\
.73\end{array}$ & $\begin{array}{c}20590 \\
.6\end{array}$ & $\begin{array}{c}21785 \\
.16\end{array}$ & $\begin{array}{c}24738 \\
.85\end{array}$ & $\begin{array}{c}26141 \\
.08\end{array}$ \\
\hline Finland & $\begin{array}{c}26796 \\
.41\end{array}$ & $\begin{array}{c}27807 \\
.75\end{array}$ & $\begin{array}{c}28605 \\
.12\end{array}$ & $\begin{array}{c}29021 \\
.64\end{array}$ & $\begin{array}{c}31175 \\
.73\end{array}$ & $\begin{array}{c}32051 \\
.76\end{array}$ & $\begin{array}{c}34408 \\
.82\end{array}$ & $\begin{array}{c}37792 \\
.56\end{array}$ & $\begin{array}{c}40083 \\
.7\end{array}$ & $\begin{array}{c}38000 \\
.02\end{array}$ & $\begin{array}{c}38985 \\
.87\end{array}$ & $\begin{array}{c}40916 \\
.65\end{array}$ & $\begin{array}{c}40872 \\
.85\end{array}$ \\
\hline France & $\begin{array}{c}26106 \\
.05 \\
\end{array}$ & $\begin{array}{c}27506 \\
.16 \\
\end{array}$ & $\begin{array}{c}28528 \\
.16 \\
\end{array}$ & $\begin{array}{c}28148 \\
.12 \\
\end{array}$ & $\begin{array}{c}29040 \\
.97 \\
\end{array}$ & $\begin{array}{c}30504 \\
.06 \\
\end{array}$ & $\begin{array}{c}32431 \\
.44 \\
\end{array}$ & $\begin{array}{c}34086 \\
.96 \\
\end{array}$ & $\begin{array}{c}35102 \\
.87 \\
\end{array}$ & $\begin{array}{c}34711 \\
.66 \\
\end{array}$ & $\begin{array}{c}35939 \\
.46 \\
\end{array}$ & $\begin{array}{c}37447 \\
.95 \\
\end{array}$ & $\begin{array}{c}37684 \\
.2 \\
\end{array}$ \\
\hline $\begin{array}{c}\text { German } \\
y\end{array}$ & $\begin{array}{c}27462 \\
.91\end{array}$ & $\begin{array}{c}28670 \\
.79\end{array}$ & $\begin{array}{c}29504 \\
.29\end{array}$ & $\begin{array}{c}30238 \\
.27\end{array}$ & $\begin{array}{c}31715 \\
.39\end{array}$ & $\begin{array}{c}32236 \\
.74\end{array}$ & $\begin{array}{c}34620 \\
.41\end{array}$ & $\begin{array}{c}36813 \\
.71\end{array}$ & $\begin{array}{c}38432 \\
.45\end{array}$ & $\begin{array}{c}37492 \\
.65\end{array}$ & $\begin{array}{c}39707 \\
.33\end{array}$ & $\begin{array}{c}42541 \\
.51\end{array}$ & $\begin{array}{c}43359 \\
.54\end{array}$ \\
\hline Greece & $\begin{array}{c}19524 \\
.7\end{array}$ & $\begin{array}{c}20963 \\
.82\end{array}$ & $\begin{array}{c}22615 \\
.96\end{array}$ & $\begin{array}{c}23871 \\
.92\end{array}$ & $\begin{array}{c}25439 \\
.32\end{array}$ & $\begin{array}{c}25577 \\
.45\end{array}$ & $\begin{array}{c}28510 \\
.88\end{array}$ & $\begin{array}{c}29284 \\
.61\end{array}$ & $\begin{array}{c}30855 \\
.94\end{array}$ & $\begin{array}{c}30381 \\
.48\end{array}$ & $\begin{array}{c}27934 \\
.32\end{array}$ & $\begin{array}{c}25671 \\
.29\end{array}$ & $\begin{array}{c}24912 \\
.13\end{array}$ \\
\hline Hungary & $\begin{array}{c}11858 \\
.33\end{array}$ & $\begin{array}{c}13213 \\
.64\end{array}$ & $\begin{array}{c}14526 \\
.39\end{array}$ & $\begin{array}{c}15465 \\
.37\end{array}$ & $\begin{array}{c}16230 \\
.26\end{array}$ & $\begin{array}{c}17073 \\
.55\end{array}$ & $\begin{array}{c}18337 \\
.21\end{array}$ & $\begin{array}{c}19041 \\
.09\end{array}$ & $\begin{array}{c}20696 \\
.73\end{array}$ & $\begin{array}{c}20689 \\
.05\end{array}$ & $\begin{array}{c}21716 \\
.65\end{array}$ & $\begin{array}{c}22999 \\
.83\end{array}$ & $\begin{array}{c}23206 \\
.26\end{array}$ \\
\hline Iceland & $\begin{array}{c}29792 \\
.56\end{array}$ & $\begin{array}{c}31875 \\
.05\end{array}$ & $\begin{array}{c}32598 \\
.38\end{array}$ & $\begin{array}{c}32690 \\
.5\end{array}$ & $\begin{array}{c}35522 \\
.71\end{array}$ & $\begin{array}{c}37427 \\
.93\end{array}$ & $\begin{array}{c}39579 \\
.74\end{array}$ & $\begin{array}{c}41435 \\
.18\end{array}$ & $\begin{array}{c}43456 \\
.22\end{array}$ & 41803 & $\begin{array}{c}39810 \\
.8\end{array}$ & $\begin{array}{c}40938 \\
.66\end{array}$ & $\begin{array}{c}42006 \\
.54\end{array}$ \\
\hline Ireland & $\begin{array}{c}30199 \\
.65 \\
\end{array}$ & $\begin{array}{c}32580 \\
.73 \\
\end{array}$ & $\begin{array}{c}35212 \\
.04 \\
\end{array}$ & $\begin{array}{c}36230 \\
.74 \\
\end{array}$ & $\begin{array}{c}38711 \\
.71 \\
\end{array}$ & $\begin{array}{c}40447 \\
.51\end{array}$ & $\begin{array}{c}44211 \\
.66\end{array}$ & $\begin{array}{c}46717 \\
.77 \\
\end{array}$ & $\begin{array}{c}44184 \\
.55\end{array}$ & $\begin{array}{c}41532 \\
.21\end{array}$ & $\begin{array}{c}43320 \\
.05 \\
\end{array}$ & 44903 & $\begin{array}{c}46274 \\
.52 \\
\end{array}$ \\
\hline Israel & $\begin{array}{c}24939 \\
.69\end{array}$ & $\begin{array}{c}24906 \\
.78\end{array}$ & $\begin{array}{c}25204 \\
.73\end{array}$ & $\begin{array}{c}23800 \\
.65\end{array}$ & $\begin{array}{c}25152 \\
.87\end{array}$ & $\begin{array}{c}24743 \\
.43\end{array}$ & $\begin{array}{c}25580 \\
.83\end{array}$ & $\begin{array}{c}27352 \\
.81\end{array}$ & $\begin{array}{c}27304 \\
.81\end{array}$ & $\begin{array}{c}27504 \\
.02\end{array}$ & $\begin{array}{c}28836 \\
.16\end{array}$ & $\begin{array}{c}30497 \\
.57\end{array}$ & $\begin{array}{c}31706 \\
.19\end{array}$ \\
\hline Italy & $\begin{array}{c}27084 \\
.2\end{array}$ & $\begin{array}{c}28043 \\
.05\end{array}$ & $\begin{array}{c}28716 \\
.2\end{array}$ & $\begin{array}{c}29116 \\
.35\end{array}$ & $\begin{array}{c}29448 \\
.8\end{array}$ & $\begin{array}{c}30016 \\
.16\end{array}$ & $\begin{array}{c}32252 \\
.42\end{array}$ & $\begin{array}{c}33897 \\
.28\end{array}$ & $\begin{array}{c}35274 \\
.31\end{array}$ & $\begin{array}{c}34347 \\
.7\end{array}$ & $\begin{array}{c}34860 \\
.58\end{array}$ & $\begin{array}{c}36183 \\
.32\end{array}$ & $\begin{array}{c}36002 \\
.91\end{array}$ \\
\hline Japan & $\begin{array}{c}26841 \\
.23\end{array}$ & $\begin{array}{c}27476 \\
.22\end{array}$ & $\begin{array}{c}28170 \\
.05\end{array}$ & $\begin{array}{c}28943 \\
.72\end{array}$ & $\begin{array}{c}30354 \\
.46\end{array}$ & $\begin{array}{c}31667 \\
.91\end{array}$ & $\begin{array}{c}33094 \\
.34\end{array}$ & $\begin{array}{c}34507 \\
.17\end{array}$ & $\begin{array}{c}34803 \\
.66\end{array}$ & $\begin{array}{c}33201 \\
.99\end{array}$ & $\begin{array}{c}34994 \\
.37\end{array}$ & $\begin{array}{c}35775 \\
.26\end{array}$ & $\begin{array}{c}37213 \\
.84\end{array}$ \\
\hline & $\begin{array}{c}18539 \\
.42\end{array}$ & $\begin{array}{c}19713 \\
.6 \\
\end{array}$ & $\begin{array}{c}21396 \\
.72 \\
\end{array}$ & $\begin{array}{c}22084 \\
.78 \\
\end{array}$ & $\begin{array}{c}23763 \\
.31 \\
\end{array}$ & $\begin{array}{c}25186 \\
.64\end{array}$ & $\begin{array}{c}26875 \\
.23 \\
\end{array}$ & $\begin{array}{c}29050 \\
.16\end{array}$ & $\begin{array}{c}29945 \\
.9 \\
\end{array}$ & $\begin{array}{c}29529 \\
.79 \\
\end{array}$ & $\begin{array}{c}31748 \\
.35\end{array}$ & $\begin{array}{c}32546 \\
.52\end{array}$ & $\begin{array}{c}33557 \\
.03 \\
\end{array}$ \\
\hline Latvia & $\begin{array}{c}8033 . \\
097\end{array}$ & $\begin{array}{c}9034 . \\
533\end{array}$ & $\begin{array}{c}10067 \\
.8\end{array}$ & $\begin{array}{c}11027 \\
.78\end{array}$ & $\begin{array}{c}12240 \\
.34\end{array}$ & $\begin{array}{c}13877 \\
.98\end{array}$ & $\begin{array}{c}15797 \\
.29\end{array}$ & $\begin{array}{c}18179 \\
.67\end{array}$ & $\begin{array}{c}19516 \\
.02\end{array}$ & $\begin{array}{c}16973 \\
.37\end{array}$ & $\begin{array}{c}17658 \\
.75\end{array}$ & $\begin{array}{c}19887 \\
.75\end{array}$ & $\begin{array}{c}21414 \\
.57\end{array}$ \\
\hline $\begin{array}{c}\text { Lithuani } \\
\text { a }\end{array}$ & $\begin{array}{c}8449 . \\
658\end{array}$ & $\begin{array}{c}9440 . \\
144\end{array}$ & $\begin{array}{c}10480 \\
.72\end{array}$ & $\begin{array}{c}12055 \\
.24\end{array}$ & $\begin{array}{c}13019 \\
.58\end{array}$ & $\begin{array}{c}14510 \\
.65\end{array}$ & $\begin{array}{c}16461 \\
.79\end{array}$ & $\begin{array}{c}19071 \\
.45\end{array}$ & $\begin{array}{c}20721 \\
.03\end{array}$ & $\begin{array}{c}18130 \\
.05\end{array}$ & $\begin{array}{c}20112 \\
.1\end{array}$ & $\begin{array}{c}22884 \\
.8\end{array}$ & $\begin{array}{c}24703 \\
.7\end{array}$ \\
\hline $\begin{array}{c}\text { Luxemb } \\
\text { ourg }\end{array}$ & $\begin{array}{c}55279 \\
.77\end{array}$ & 55853 & $\begin{array}{c}58709 \\
.02\end{array}$ & $\begin{array}{c}59960 \\
.03\end{array}$ & $\begin{array}{c}63998 \\
.59\end{array}$ & $\begin{array}{c}68140 \\
.65\end{array}$ & $\begin{array}{c}77880 \\
.53\end{array}$ & $\begin{array}{c}83837 \\
.45\end{array}$ & $\begin{array}{c}86591 \\
.99\end{array}$ & 82250 & $\begin{array}{c}85587 \\
.01\end{array}$ & $\begin{array}{c}91814 \\
.04\end{array}$ & $\begin{array}{c}91526 \\
.72\end{array}$ \\
\hline Mexico & $\begin{array}{c}10870 \\
.41\end{array}$ & $\begin{array}{c}10931 \\
.09\end{array}$ & $\begin{array}{c}10999 \\
.98\end{array}$ & $\begin{array}{c}11302 \\
.74\end{array}$ & $\begin{array}{c}11921 \\
.84\end{array}$ & $\begin{array}{c}12539 \\
.96\end{array}$ & $\begin{array}{c}13720 \\
.69\end{array}$ & $\begin{array}{c}14233 \\
.03\end{array}$ & $\begin{array}{c}14885 \\
.9\end{array}$ & $\begin{array}{c}14532 \\
.39\end{array}$ & $\begin{array}{c}15257 \\
.67\end{array}$ & $\begin{array}{c}16546 \\
.64\end{array}$ & $\begin{array}{c}17220 \\
.07\end{array}$ \\
\hline $\begin{array}{l}\text { Netherla } \\
\text { nds }\end{array}$ & $\begin{array}{c}31883 \\
.7\end{array}$ & $\begin{array}{c}33194 \\
.9\end{array}$ & $\begin{array}{c}34447 \\
.05\end{array}$ & $\begin{array}{c}34115 \\
.07\end{array}$ & $\begin{array}{c}35779 \\
.62\end{array}$ & $\begin{array}{c}37625 \\
.25\end{array}$ & $\begin{array}{c}40959 \\
.19\end{array}$ & $\begin{array}{c}43885 \\
.02\end{array}$ & $\begin{array}{c}46419 \\
.04\end{array}$ & $\begin{array}{c}44590 \\
.78\end{array}$ & $\begin{array}{c}45079 \\
.37\end{array}$ & $\begin{array}{c}46599 \\
.23\end{array}$ & $\begin{array}{c}47271 \\
.97\end{array}$ \\
\hline $\begin{array}{c}\text { New } \\
\text { Zealand }\end{array}$ & $\begin{array}{c}21471 \\
.62\end{array}$ & $\begin{array}{c}22387 \\
.15\end{array}$ & $\begin{array}{c}23180 \\
.06\end{array}$ & $\begin{array}{c}23856 \\
.57\end{array}$ & $\begin{array}{c}24995 \\
.65\end{array}$ & $\begin{array}{c}25590 \\
.13\end{array}$ & $\begin{array}{c}27682 \\
.12\end{array}$ & $\begin{array}{c}29304 \\
.03\end{array}$ & $\begin{array}{c}29784 \\
.43\end{array}$ & $\begin{array}{c}30587 \\
.84\end{array}$ & $\begin{array}{c}31174 \\
.16\end{array}$ & $\begin{array}{c}32680 \\
.39\end{array}$ & $\begin{array}{c}32924 \\
.63 \\
\end{array}$ \\
\hline Norway & $\begin{array}{c}36952 \\
.28\end{array}$ & $\begin{array}{c}37778 \\
.56\end{array}$ & $\begin{array}{c}37981 \\
.69 \\
\end{array}$ & $\begin{array}{c}38552 \\
.71 \\
\end{array}$ & $\begin{array}{c}42513 \\
.85\end{array}$ & $\begin{array}{c}47800 \\
.54\end{array}$ & $\begin{array}{c}54084 \\
.29\end{array}$ & $\begin{array}{c}55864 \\
.47 \\
\end{array}$ & $\begin{array}{c}61719 \\
.34 \\
\end{array}$ & $\begin{array}{c}55401 \\
.83 \\
\end{array}$ & $\begin{array}{c}57966 \\
.87 \\
\end{array}$ & $\begin{array}{c}62077 \\
.85 \\
\end{array}$ & $\begin{array}{c}65349 \\
.22 \\
\end{array}$ \\
\hline Poland & $\begin{array}{c}10677 \\
.5\end{array}$ & $\begin{array}{c}11123 \\
.67\end{array}$ & $\begin{array}{c}11803 \\
.76\end{array}$ & $\begin{array}{c}12278 \\
.87\end{array}$ & $\begin{array}{c}13343 \\
.39\end{array}$ & $\begin{array}{c}13898 \\
.41\end{array}$ & $\begin{array}{c}15135 \\
.75\end{array}$ & $\begin{array}{c}16784 \\
.45\end{array}$ & $\begin{array}{c}18308 \\
.04\end{array}$ & $\begin{array}{c}19088 \\
.29\end{array}$ & $\begin{array}{c}20828 \\
.99\end{array}$ & $\begin{array}{c}22553 \\
.51\end{array}$ & $\begin{array}{c}23455 \\
.56\end{array}$ \\
\hline Portugal & $\begin{array}{c}18884 \\
.87 \\
\end{array}$ & $\begin{array}{c}19533 \\
.39 \\
\end{array}$ & $\begin{array}{c}20356 \\
.72 \\
\end{array}$ & $\begin{array}{c}20829 \\
.64 \\
\end{array}$ & $\begin{array}{c}21459 \\
.95 \\
\end{array}$ & $\begin{array}{c}22725 \\
.35 \\
\end{array}$ & $\begin{array}{c}24649 \\
.68 \\
\end{array}$ & $\begin{array}{c}25701 \\
.79 \\
\end{array}$ & $\begin{array}{c}26665 \\
.77 \\
\end{array}$ & $\begin{array}{c}26478 \\
.49 \\
\end{array}$ & $\begin{array}{c}27282 \\
.77 \\
\end{array}$ & $\begin{array}{c}26769 \\
.43 \\
\end{array}$ & $\begin{array}{c}26438 \\
.14 \\
\end{array}$ \\
\hline $\begin{array}{c}\text { Slovak } \\
\text { Republi } \\
\text { c } \\
\end{array}$ & $\begin{array}{c}11356 \\
.25\end{array}$ & $\begin{array}{c}12389 \\
.81\end{array}$ & $\begin{array}{c}13307 \\
.88\end{array}$ & $\begin{array}{c}14150 \\
.81\end{array}$ & $\begin{array}{c}15185 \\
.21\end{array}$ & $\begin{array}{c}16595 \\
.26\end{array}$ & $\begin{array}{c}18826 \\
.14\end{array}$ & $\begin{array}{c}21110 \\
.42\end{array}$ & $\begin{array}{c}23608 \\
.98\end{array}$ & $\begin{array}{c}22963 \\
.91\end{array}$ & $\begin{array}{c}25030 \\
.42\end{array}$ & $\begin{array}{c}26084 \\
.11\end{array}$ & $\begin{array}{c}26973 \\
.79 \\
\end{array}$ \\
\hline Slovenia & $\begin{array}{c}18004 \\
.8\end{array}$ & $\begin{array}{c}18957 \\
.42\end{array}$ & $\begin{array}{c}20236 \\
.43\end{array}$ & $\begin{array}{c}21084 \\
.86\end{array}$ & $\begin{array}{c}22739 \\
.14\end{array}$ & $\begin{array}{c}23848 \\
.6\end{array}$ & $\begin{array}{c}25673 \\
.49\end{array}$ & $\begin{array}{c}27526 \\
.54\end{array}$ & $\begin{array}{c}29594 \\
.67\end{array}$ & $\begin{array}{c}27531 \\
.13\end{array}$ & $\begin{array}{c}27844 \\
.9\end{array}$ & $\begin{array}{c}28931 \\
.42\end{array}$ & $\begin{array}{c}29048 \\
.33\end{array}$ \\
\hline Spain & $\begin{array}{c}21600 \\
.82 \\
\end{array}$ & $\begin{array}{c}23006 \\
.61 \\
\end{array}$ & $\begin{array}{c}24376 \\
.33 \\
\end{array}$ & $\begin{array}{c}25015 \\
.22 \\
\end{array}$ & $\begin{array}{c}26160 \\
.19 \\
\end{array}$ & $\begin{array}{c}27600 \\
.94 \\
\end{array}$ & $\begin{array}{c}30704 \\
.58 \\
\end{array}$ & $\begin{array}{c}32423 \\
.53 \\
\end{array}$ & $\begin{array}{c}33242 \\
.25 \\
\end{array}$ & $\begin{array}{c}32112 \\
.82 \\
\end{array}$ & $\begin{array}{c}31716 \\
.71 \\
\end{array}$ & $\begin{array}{c}31872 \\
.36 \\
\end{array}$ & $\begin{array}{c}31724 \\
.63 \\
\end{array}$ \\
\hline Sweden & $\begin{array}{c}29631 \\
.19 \\
\end{array}$ & $\begin{array}{c}29940 \\
.41 \\
\end{array}$ & $\begin{array}{c}30926 \\
.36 \\
\end{array}$ & $\begin{array}{c}31782 \\
.18 \\
\end{array}$ & $\begin{array}{c}33830 \\
.67 \\
\end{array}$ & $\begin{array}{c}34244 \\
.36\end{array}$ & $\begin{array}{c}37679 \\
.72 \\
\end{array}$ & $\begin{array}{c}40855 \\
.47 \\
\end{array}$ & $\begin{array}{c}42158 \\
.47 \\
\end{array}$ & $\begin{array}{c}40309 \\
.18 \\
\end{array}$ & $\begin{array}{c}42256 \\
.28 \\
\end{array}$ & $\begin{array}{c}44608 \\
.64\end{array}$ & $\begin{array}{c}45432 \\
.31 \\
\end{array}$ \\
\hline
\end{tabular}




\begin{tabular}{|c|c|c|c|c|c|c|c|c|c|c|c|c|c|}
\hline $\begin{array}{c}\text { United } \\
\text { Kingdo } \\
\mathrm{m}\end{array}$ & $\begin{array}{c}26423 \\
.95\end{array}$ & $\begin{array}{c}27733 \\
.45\end{array}$ & $\begin{array}{c}28999 \\
.26\end{array}$ & $\begin{array}{c}30238 \\
.34\end{array}$ & $\begin{array}{c}31922 \\
.8\end{array}$ & $\begin{array}{c}32586 \\
.15\end{array}$ & $\begin{array}{c}34668 \\
.8\end{array}$ & $\begin{array}{c}35507 \\
.37\end{array}$ & $\begin{array}{c}36634 \\
.61\end{array}$ & $\begin{array}{c}35052 \\
.17\end{array}$ & $\begin{array}{c}36468 \\
.17\end{array}$ & $\begin{array}{c}37154 \\
.16\end{array}$ & $\begin{array}{c}38296 \\
.7\end{array}$ \\
\hline $\begin{array}{c}\text { United } \\
\text { States }\end{array}$ & 36304 & 37099 & 37979 & 39426 & 41647 & 44043 & 46230 & 47902 & 48311 & 47028 & 48396 & 49814 & 51548 \\
& .91 & .61 & .09 & .84 & .73 & .85 & .06 & .22 & .16 & .74 & .37 & .02 \\
\hline
\end{tabular}

\begin{tabular}{|c|c|c|c|c|c|c|c|}
\hline & 2013 & 2014 & 2015 & 2016 & 2017 & 2018 & 2019 \\
\hline & & & & & & & \\
\hline Australia & 47761.21 & 47644.76 & 47317.25 & 50237.79 & 50853.62 & 53061.63 & 53067.88 \\
\hline Austria & 47936.68 & 48813.53 & 49942.06 & 52665.09 & 54185.34 & 57068.53 & 58664.72 \\
\hline Belgium & 43672.71 & 44929.93 & 46201.69 & 48599.2 & 50436.26 & 52668.41 & 54693.24 \\
\hline Canada & 44298.51 & 45753.78 & 44670.05 & 46472.37 & 48319.72 & 50250.04 & 50666.14 \\
\hline Chile & 22352.53 & 22687.93 & 22603.77 & 23349.68 & 24402.91 & 24743.08 & 26128.4 \\
\hline $\begin{array}{c}\text { Czech } \\
\text { Republic }\end{array}$ & 30828.53 & 32504.22 & 33909.31 & 36101.29 & 38842.9 & 41147.63 & 43015.81 \\
\hline Denmark & 46742.94 & 47905.48 & 49058.14 & 51967.02 & 55356.49 & 57459.17 & 60308.07 \\
\hline Estonia & 27596.46 & 29107.89 & 29436.42 & 31574.45 & 33902.1 & 36406.05 & 38880.85 \\
\hline Finland & 41492.92 & 41749.86 & 42490.21 & 44934.49 & 47570.27 & 49726.71 & 51620.97 \\
\hline France & 39528.47 & 40144.06 & 40829.89 & 42855.94 & 44480.72 & 46456.11 & 49225.56 \\
\hline Germany & 44993.67 & 47011.28 & 47609.56 & 50579.48 & 52952.9 & 54954.69 & 55891.16 \\
\hline Greece & 25947.87 & 26642.23 & 26720.91 & 27471.22 & 28644.98 & 29680.9 & 30869.25 \\
\hline Hungary & 24498.66 & 25642.56 & 26777.47 & 27911.66 & 29465.13 & 31829.55 & 33949.74 \\
\hline Iceland & 44404.73 & 45995 & 49203.29 & 53486.62 & 55648.25 & 58139.72 & 59567.24 \\
\hline Ireland & 47924.39 & 51212.5 & 69133.71 & 71793.29 & 78739.39 & 85027 & 89561.46 \\
\hline
\end{tabular}

\begin{tabular}{|c|c|c|c|c|c|c|c|}
\hline Israel & 34167.04 & 34282.24 & 35487.89 & 37806.43 & 38849.91 & 40351.27 & 41964.74 \\
\hline Italy & 36067.71 & 36194.92 & 36899.4 & 39926.97 & 41581.13 & 43085.03 & 44367.94 \\
\hline Japan & 39008.36 & 39183.47 & 40398.4 & 39983.64 & 40992.39 & 41724.03 & 42385.77 \\
\hline South Korea & 34244.24 & 35324.26 & 37902.36 & 39575.3 & 40957.35 & 42486.74 & 42728.03 \\
\hline Latvia & 22811.03 & 23798.23 & 24964.05 & 26712.84 & 28666 & 30814.22 & 32057.95 \\
\hline Lithuania & 26721.6 & 28184.5 & 28834.46 & 30925.15 & 33761.91 & 36346.42 & 38756.14 \\
\hline Luxembourg & 95246.11 & 100933.6 & 103760.1 & 110260.9 & 112187.7 & 116480.8 & 120670.5 \\
\hline Mexico & 17461.8 & 18178.4 & 18454.8 & 19516.15 & 19947.2 & 20519.42 & 20741.1 \\
\hline Netherlands & 49242.79 & 49233.23 & 50288.35 & 52289.4 & 55089.58 & 57899.82 & 59468.67 \\
\hline New Zealand & 36100.25 & 37133.67 & 37338.64 & 39896.68 & 42208.48 & 43376.97 & 44152.06 \\
\hline Norway & 66956.29 & 65895.67 & 60352.72 & 58923.24 & 64050.37 & 69709.38 & 68343.74 \\
\hline Poland & 24272.84 & 25162.88 & 26534.72 & 27985.34 & 29714.59 & 31613.69 & 33774.13 \\
\hline Portugal & 27936.01 & 28742.31 & 29660.85 & 31607.61 & 33044.7 & 34931.86 & 36760.02 \\
\hline
\end{tabular}




\begin{tabular}{|c|c|c|c|c|c|c|c|}
\hline Slovak Republic & 28004.53 & 28997.38 & 29927.68 & 29659.33 & 30097.99 & 31561.92 & 32550.32 \\
\hline Slovenia & 29979.62 & 30872.73 & 31631.84 & 33942.77 & 36515.7 & 38952.06 & 41181.43 \\
\hline Spain & 32452.66 & 33544.35 & 34929.21 & 37313.63 & 39580.19 & 40780.29 & 42211.81 \\
\hline Sweden & 46312.29 & 47184.72 & 49103.06 & 50430.17 & 51947.94 & 53553.31 & 55068.77 \\
\hline United Kingdom & 39945.1 & 41292.49 & 42571.65 & 44125.53 & 45757.78 & 47163.17 & 48542.09 \\
\hline United States & 53056.68 & 55008.01 & 56831.65 & 58000.89 & 60091.57 & 63043.05 & 65240.38 \\
\hline
\end{tabular}

Source: OECD, GDP Database: https://stats.oecd.org/ 
Table $2 \mathrm{~b}$

Historical average GDP rate of OECD Countries

\begin{tabular}{|c|c|c|c|c|c|c|c|c|c|c|c|c|c|}
\hline Year & 2000 & 2001 & 2002 & 2003 & 2004 & 2005 & 2006 & 2007 & 2008 & 2009 & 2010 & 2011 & 2012 \\
\hline $\begin{array}{c}\text { GDP } \\
\mathrm{h}(\%)\end{array}$ & $\begin{array}{c}3.99 \\
\%\end{array}$ & $\begin{array}{c}1.41 \\
\%\end{array}$ & $\begin{array}{c}1.55 \\
\%\end{array}$ & $\begin{array}{c}2.05 \\
\%\end{array}$ & $\begin{array}{c}3.22 \\
\%\end{array}$ & $\begin{array}{c}2.84 \\
\%\end{array}$ & $\begin{array}{c}3.10 \\
\%\end{array}$ & $\begin{array}{c}2.73 \\
\%\end{array}$ & $\begin{array}{c}0.27 \\
\%\end{array}$ & $\begin{array}{c}3.44 \\
\%\end{array}$ & $\begin{array}{c}2.94 \\
\%\end{array}$ & $\begin{array}{c}1.85 \\
\%\end{array}$ & $\begin{array}{c}1.28 \\
\%\end{array}$ \\
\hline $\begin{array}{c}\text { Annual } \\
\text { Change }\end{array}$ & $\begin{array}{c}0.81 \\
\%\end{array}$ & $\begin{array}{c}2.58 \\
\%\end{array}$ & $\begin{array}{c}0.14 \\
\%\end{array}$ & $\begin{array}{c}0.51 \\
\%\end{array}$ & $\begin{array}{c}1.17 \\
\%\end{array}$ & $\begin{array}{c}0.38 \\
\%\end{array}$ & $\begin{array}{c}0.26 \\
\%\end{array}$ & $\begin{array}{c}0.37 \\
\%\end{array}$ & $\begin{array}{c}2.46 \\
\%\end{array}$ & $\begin{array}{c}3.71 \\
\%\end{array}$ & $\begin{array}{c}6.38 \\
\%\end{array}$ & $\begin{array}{c}1.09 \\
\%\end{array}$ & $\begin{array}{c}0.57 \\
\%\end{array}$ \\
\hline
\end{tabular}

\begin{tabular}{|c|c|c|c|c|c|c|c|}
\hline Year & 2013 & 2014 & 2015 & 2016 & 2017 & 2018 & 2019 \\
\hline GDP Growth (\%) & $1.51 \%$ & $2.07 \%$ & $2.42 \%$ & $1.75 \%$ & $2.57 \%$ & $2.21 \%$ & $1.63 \%$ \\
\hline Annual Change & $0.23 \%$ & $0.56 \%$ & $0.35 \%$ & $-0.67 \%$ & $0.82 \%$ & $-0.36 \%$ & $-0.58 \%$ \\
\hline
\end{tabular}

Source: Macrotrends, GDP Rate Database: https://www.macrotrends.net/countries/OED/oecd-members/gdpgrowth-rate

Table 3a

Unemployment rate of 34 OECD countries

\begin{tabular}{|c|c|c|c|c|c|c|c|c|c|c|c|c|c|}
\hline & 2000 & 2001 & 2002 & 2003 & 2004 & 2005 & 2006 & 2007 & 2008 & 2009 & 2010 & 2011 & 2012 \\
\hline $\begin{array}{c}\text { Austr } \\
\text { alia }\end{array}$ & $\begin{array}{c}6.285 \\
546 \\
\end{array}$ & $\begin{array}{c}6.742 \\
173 \\
\end{array}$ & $\begin{array}{c}6.368 \\
911 \\
\end{array}$ & $\begin{array}{c}5.928 \\
42 \\
\end{array}$ & $\begin{array}{c}5.396 \\
734 \\
\end{array}$ & $\begin{array}{c}5.033 \\
881 \\
\end{array}$ & $\begin{array}{c}4.785 \\
24 \\
\end{array}$ & $\begin{array}{c}4.379 \\
151 \\
\end{array}$ & $\begin{array}{c}4.234 \\
33 \\
\end{array}$ & $\begin{array}{c}5.560 \\
385 \\
\end{array}$ & $\begin{array}{c}5.213 \\
34\end{array}$ & $\begin{array}{c}5.081 \\
195\end{array}$ & $\begin{array}{c}5.223 \\
376\end{array}$ \\
\hline $\begin{array}{c}\text { Austri } \\
\text { a }\end{array}$ & $\begin{array}{c}3.933 \\
333\end{array}$ & $\begin{array}{c}3.983 \\
333\end{array}$ & $\begin{array}{c}4.383 \\
333\end{array}$ & $\begin{array}{c}4.758 \\
333\end{array}$ & $\begin{array}{c}5.508 \\
333\end{array}$ & $\begin{array}{c}5.633 \\
333\end{array}$ & 5.25 & $\begin{array}{c}4.866 \\
667\end{array}$ & $\begin{array}{c}4.141 \\
667\end{array}$ & 5.325 & $\begin{array}{c}4.841 \\
667\end{array}$ & 4.575 & $\begin{array}{c}4.883 \\
333\end{array}$ \\
\hline $\begin{array}{c}\text { Belgi } \\
\text { um }\end{array}$ & 6.875 & $\begin{array}{c}6.591 \\
667\end{array}$ & 7.525 & 8.175 & $\begin{array}{c}8.391 \\
666\end{array}$ & $\begin{array}{c}8.433 \\
333\end{array}$ & 8.25 & $\begin{array}{c}7.483 \\
333\end{array}$ & 6.975 & $\begin{array}{c}7.891 \\
667\end{array}$ & $\begin{array}{c}8.316 \\
667\end{array}$ & $\begin{array}{c}7.166 \\
667\end{array}$ & $\begin{array}{c}7.566 \\
667\end{array}$ \\
\hline $\begin{array}{c}\text { Canad } \\
\text { a }\end{array}$ & $\begin{array}{c}6.816 \\
667\end{array}$ & $\begin{array}{c}7.216 \\
667\end{array}$ & 7.675 & 7.575 & $\begin{array}{c}7.191 \\
667\end{array}$ & $\begin{array}{c}6.758 \\
333\end{array}$ & 6.35 & $\begin{array}{c}6.066 \\
667\end{array}$ & 6.175 & 8.375 & $\begin{array}{c}8.116 \\
667\end{array}$ & $\begin{array}{c}7.558 \\
333\end{array}$ & $\begin{array}{c}7.333 \\
333\end{array}$ \\
\hline Chile & $\begin{array}{c}9.667 \\
5\end{array}$ & $\begin{array}{c}9.852 \\
5\end{array}$ & 9.805 & 9.54 & $\begin{array}{c}10.00 \\
75\end{array}$ & 9.235 & 7.8 & 7.13 & $\begin{array}{c}7.792 \\
5\end{array}$ & 9.675 & 8.15 & 7.125 & $\begin{array}{c}6.545 \\
561\end{array}$ \\
\hline $\begin{array}{c}\text { Czech } \\
\text { Repub } \\
\text { lic }\end{array}$ & 8.775 & 8.125 & $\begin{array}{c}7.291 \\
667\end{array}$ & 7.775 & $\begin{array}{c}8.291 \\
667\end{array}$ & $\begin{array}{c}7.941 \\
667\end{array}$ & $\begin{array}{c}7.133 \\
333\end{array}$ & 5.325 & $\begin{array}{c}4.408 \\
333\end{array}$ & $\begin{array}{c}6.683 \\
333\end{array}$ & $\begin{array}{c}7.291 \\
667\end{array}$ & $\begin{array}{c}6.716 \\
667\end{array}$ & 6.975 \\
\hline $\begin{array}{c}\text { Denm } \\
\text { ark }\end{array}$ & 4.325 & 4.525 & $\begin{array}{c}4.583 \\
333 \\
\end{array}$ & $\begin{array}{c}5.408 \\
333\end{array}$ & 5.5 & $\begin{array}{c}4.841 \\
667 \\
\end{array}$ & $\begin{array}{c}3.908 \\
333\end{array}$ & 3.75 & $\begin{array}{c}3.708 \\
333\end{array}$ & $\begin{array}{c}6.408 \\
333 \\
\end{array}$ & $\begin{array}{c}7.766 \\
667 \\
\end{array}$ & $\begin{array}{c}7.758 \\
333\end{array}$ & 7.8 \\
\hline
\end{tabular}

\begin{tabular}{|c|c|c|c|c|c|c|c|c|c|c|c|c|c|}
\hline Estonia & $\begin{array}{c}14.45 \\
833 \\
\end{array}$ & $\begin{array}{c}13.04 \\
167 \\
\end{array}$ & $\begin{array}{c}11.33 \\
333\end{array}$ & 0.35 & $\begin{array}{c}10.08 \\
333\end{array}$ & 7.975 & $\begin{array}{c}5.941 \\
667 \\
\end{array}$ & 4.6 & $\begin{array}{c}5.516 \\
667 \\
\end{array}$ & $\begin{array}{c}13.57 \\
5 \\
\end{array}$ & $\begin{array}{c}16.74 \\
167 \\
\end{array}$ & $\begin{array}{c}12.42 \\
5\end{array}$ & $\begin{array}{c}9.991 \\
667 \\
\end{array}$ \\
\hline Finlan & $\begin{array}{c}9.783 \\
334 \\
\end{array}$ & 125 & 9.075 & & 3.825 & 24 & $\begin{array}{c}7.716 \\
667 \\
\end{array}$ & & $\begin{array}{c}6.366 \\
667 \\
\end{array}$ & $\begin{array}{c}8.241 \\
667\end{array}$ & $\begin{array}{c}8.383 \\
333 \\
\end{array}$ & 7.775 & $\begin{array}{c}7.683 \\
333 \\
\end{array}$ \\
\hline France & $\begin{array}{c}9.558 \\
333 \\
\end{array}$ & & & 85 & 85 & & & & 7.425 & & $\begin{array}{c}9.266 \\
666\end{array}$ & & $\begin{array}{c}9.766 \\
666 \\
\end{array}$ \\
\hline Germa & $\begin{array}{c}8.008 \\
333 \\
\end{array}$ & & & & 10.5 & & $\begin{array}{c}10.27 \\
5 \\
\end{array}$ & & 7.425 & $\begin{array}{c}7.641 \\
667 \\
\end{array}$ & $\begin{array}{c}6.966 \\
667 \\
\end{array}$ & $\begin{array}{r}5.8 \\
33 \\
\end{array}$ & $\begin{array}{c}5.383 \\
333 \\
\end{array}$ \\
\hline Greec & $\begin{array}{c}11.23 \\
333\end{array}$ & $\begin{array}{c}10.67 \\
5\end{array}$ & & & & & $\begin{array}{c}9.033 \\
334\end{array}$ & & $\begin{array}{c}7.766 \\
667\end{array}$ & 9.625 & 1275 & & $\begin{array}{c}24.49 \\
167\end{array}$ \\
\hline & $\begin{array}{c}6.266 \\
667\end{array}$ & 5.575 & & & & 7175 & $\begin{array}{c}7.458 \\
333\end{array}$ & $\begin{array}{c}7.391 \\
667\end{array}$ & 7.8 & 9.675 & 10.8 & & $\begin{array}{c}10.67 \\
5\end{array}$ \\
\hline Iceland & & $\begin{array}{c}3.408 \\
333\end{array}$ & $\begin{array}{c}2.883 \\
333\end{array}$ & $\begin{array}{c}3.208 \\
333\end{array}$ & 2.475 & & $\begin{array}{c}8.016 \\
666\end{array}$ & 8.3 & J & $\begin{array}{c}6.633 \\
333\end{array}$ & $\begin{array}{c}5.841 \\
667\end{array}$ & $\begin{array}{c}5.408 \\
333\end{array}$ & $\begin{array}{c}4.508 \\
333\end{array}$ \\
\hline Ireland & 4.5 & 4.175 & 4.725 & 4.85 & $\begin{array}{c}4.741 \\
667\end{array}$ & & $\begin{array}{c}4.783 \\
333\end{array}$ & 5 & $\begin{array}{c}6.783 \\
333\end{array}$ & 12.65 & $\begin{array}{c}14.55 \\
833\end{array}$ & $\begin{array}{c}15.40 \\
833\end{array}$ & $\begin{array}{c}15.49 \\
167\end{array}$ \\
\hline
\end{tabular}




\begin{tabular}{|c|c|c|c|c|c|c|c|c|c|c|c|c|c|}
\hline Israel & $\begin{array}{c}8.770 \\
606\end{array}$ & $\begin{array}{c}9.348 \\
134\end{array}$ & $\begin{array}{c}10.29 \\
844\end{array}$ & $\begin{array}{c}10.71 \\
266\end{array}$ & $\begin{array}{c}10.36 \\
771\end{array}$ & $\begin{array}{c}8.989 \\
903\end{array}$ & $\begin{array}{c}8.403 \\
837\end{array}$ & $\begin{array}{c}7.318 \\
558\end{array}$ & $\begin{array}{c}6.099 \\
141\end{array}$ & $\begin{array}{c}7.544 \\
086\end{array}$ & $\begin{array}{c}6.636 \\
595\end{array}$ & $\begin{array}{c}5.602 \\
376\end{array}$ & 6.85 \\
\hline Italy & 10.05 & $\begin{array}{c}9.008 \\
333\end{array}$ & 8.475 & $\begin{array}{c}8.433 \\
333\end{array}$ & 8 & $\begin{array}{c}7.708 \\
333\end{array}$ & $\begin{array}{c}6.791 \\
667\end{array}$ & 6.075 & $\begin{array}{c}6.708 \\
333\end{array}$ & 7.75 & 8.35 & 8.35 & $\begin{array}{c}10.64 \\
167\end{array}$ \\
\hline Japan & $\begin{array}{c}4.716 \\
667\end{array}$ & $\begin{array}{c}5.033 \\
333\end{array}$ & 5.375 & $\begin{array}{c}5.258 \\
333\end{array}$ & $\begin{array}{c}4.716 \\
667\end{array}$ & 4.425 & $\begin{array}{c}4.141 \\
667\end{array}$ & $\begin{array}{c}3.841 \\
667\end{array}$ & $\begin{array}{c}3.991 \\
667\end{array}$ & $\begin{array}{c}5.066 \\
667\end{array}$ & 5.05 & $\begin{array}{c}4.583 \\
333\end{array}$ & 4.35 \\
\hline $\begin{array}{l}\text { South } \\
\text { Korea }\end{array}$ & 4.425 & 4 & $\begin{array}{c}3.258 \\
333\end{array}$ & 3.55 & $\begin{array}{c}3.658 \\
333\end{array}$ & 3.75 & 3.475 & $\begin{array}{c}3.258 \\
333\end{array}$ & 3.175 & $\begin{array}{c}3.633 \\
333\end{array}$ & $\begin{array}{c}3.708 \\
333\end{array}$ & $\begin{array}{c}3.408 \\
333\end{array}$ & 3.225 \\
\hline Latvia & $\begin{array}{c}14.34 \\
167 \\
\end{array}$ & $\begin{array}{c}13.48 \\
333 \\
\end{array}$ & $\begin{array}{c}12.49 \\
167 \\
\end{array}$ & $\begin{array}{c}11.64 \\
167 \\
\end{array}$ & 11.75 & 10.05 & $\begin{array}{c}7.041 \\
667 \\
\end{array}$ & $\begin{array}{c}6.058 \\
333 \\
\end{array}$ & $\begin{array}{c}7.741 \\
667 \\
\end{array}$ & $\begin{array}{c}17.56 \\
667 \\
\end{array}$ & $\begin{array}{c}19.47 \\
5 \\
\end{array}$ & $\begin{array}{c}16.21 \\
667 \\
\end{array}$ & $\begin{array}{c}15.04 \\
167 \\
\end{array}$ \\
\hline $\begin{array}{c}\text { Lithuani } \\
\mathrm{a} \\
\end{array}$ & $\begin{array}{c}16.44 \\
167 \\
\end{array}$ & $\begin{array}{c}17.41 \\
667 \\
\end{array}$ & $\begin{array}{c}13.77 \\
5 \\
\end{array}$ & $\begin{array}{c}12.43 \\
333 \\
\end{array}$ & $\begin{array}{c}10.89 \\
167 \\
\end{array}$ & $\begin{array}{c}8.316 \\
667 \\
\end{array}$ & $\begin{array}{c}5.791 \\
667 \\
\end{array}$ & $\begin{array}{c}4.258 \\
333 \\
\end{array}$ & 5.825 & 13.8 & $\begin{array}{c}17.84 \\
167 \\
\end{array}$ & $\begin{array}{c}15.39 \\
167 \\
\end{array}$ & $\begin{array}{c}13.40 \\
833 \\
\end{array}$ \\
\hline $\begin{array}{c}\text { Luxemb } \\
\text { ourg }\end{array}$ & $\begin{array}{c}2.233 \\
333\end{array}$ & 1.9 & $\begin{array}{c}2.558 \\
333\end{array}$ & $\begin{array}{c}3.808 \\
333\end{array}$ & 4.95 & 4.65 & 4.575 & $\begin{array}{c}4.166 \\
667\end{array}$ & $\begin{array}{c}4.916 \\
667\end{array}$ & $\begin{array}{c}5.116 \\
667\end{array}$ & $\begin{array}{c}4.583 \\
333\end{array}$ & $\begin{array}{c}4.833 \\
333\end{array}$ & $\begin{array}{c}5.066 \\
667\end{array}$ \\
\hline Mexico & $\begin{array}{c}2.505 \\
833\end{array}$ & $\begin{array}{c}2.756 \\
667\end{array}$ & $\begin{array}{c}2.978 \\
333\end{array}$ & $\begin{array}{c}3.405 \\
833\end{array}$ & $\begin{array}{l}3.915 \\
833\end{array}$ & $\begin{array}{c}3.595 \\
785\end{array}$ & $\begin{array}{c}3.596 \\
744\end{array}$ & $\begin{array}{c}3.721 \\
604\end{array}$ & $\begin{array}{c}3.974 \\
655\end{array}$ & $\begin{array}{c}5.479 \\
068\end{array}$ & $\begin{array}{c}5.380 \\
876\end{array}$ & $\begin{array}{c}5.233 \\
573\end{array}$ & $\begin{array}{c}4.952 \\
769 \\
\end{array}$ \\
\hline $\begin{array}{c}\text { Netherla } \\
\text { nds }\end{array}$ & $\begin{array}{c}3.666 \\
667 \\
\end{array}$ & 3.075 & $\begin{array}{c}3.666 \\
667 \\
\end{array}$ & $\begin{array}{c}4.841 \\
667 \\
\end{array}$ & 5.675 & $\begin{array}{c}5.883 \\
333 \\
\end{array}$ & $\begin{array}{c}5.008 \\
333 \\
\end{array}$ & 4.175 & $\begin{array}{c}3.666 \\
667 \\
\end{array}$ & 4.35 & $\begin{array}{c}5.008 \\
333\end{array}$ & $\begin{array}{c}4.991 \\
667 \\
\end{array}$ & 5.825 \\
\hline $\begin{array}{c}\text { New } \\
\text { Zealand }\end{array}$ & 6.15 & 5.45 & 5.3 & 4.775 & 4.025 & 3.825 & 3.85 & 3.575 & 4.025 & 5.875 & 6.225 & 6.025 & 6.475 \\
\hline Norway & $\begin{array}{c}3.241 \\
667\end{array}$ & $\begin{array}{c}3.408 \\
333\end{array}$ & $\begin{array}{c}3.666 \\
667\end{array}$ & $\begin{array}{c}4.208 \\
333\end{array}$ & 4.275 & 4.525 & 3.425 & $\begin{array}{c}2.558 \\
333\end{array}$ & $\begin{array}{c}2.733 \\
333\end{array}$ & $\begin{array}{c}3.283 \\
333\end{array}$ & $\begin{array}{c}3.733 \\
333\end{array}$ & $\begin{array}{c}3.408 \\
333\end{array}$ & 3.3 \\
\hline Poland & $\begin{array}{c}16.07 \\
5 \\
\end{array}$ & $\begin{array}{c}18.30 \\
833\end{array}$ & $\begin{array}{c}20.03 \\
333 \\
\end{array}$ & 19.75 & $\begin{array}{c}19.14 \\
167\end{array}$ & $\begin{array}{c}17.92 \\
5\end{array}$ & $\begin{array}{c}13.96 \\
667\end{array}$ & $\begin{array}{c}9.608 \\
334\end{array}$ & $\begin{array}{c}7.041 \\
667\end{array}$ & $\begin{array}{c}8.116 \\
667\end{array}$ & 9.675 & 9.65 & $\begin{array}{c}10.10 \\
833 \\
\end{array}$ \\
\hline Portugal & 5.075 & $\begin{array}{c}5.133 \\
333 \\
\end{array}$ & $\begin{array}{c}6.141 \\
667 \\
\end{array}$ & 7.4 & $\begin{array}{c}7.766 \\
667 \\
\end{array}$ & $\begin{array}{c}8.766 \\
666 \\
\end{array}$ & $\begin{array}{c}8.866 \\
667 \\
\end{array}$ & $\begin{array}{c}9.133 \\
333\end{array}$ & $\begin{array}{c}8.783 \\
334 \\
\end{array}$ & $\begin{array}{c}10.68 \\
333 \\
\end{array}$ & $\begin{array}{c}11.98 \\
333\end{array}$ & $\begin{array}{c}12.88 \\
333 \\
\end{array}$ & $\begin{array}{c}15.78 \\
333 \\
\end{array}$ \\
\hline $\begin{array}{c}\text { Slovak } \\
\text { Republi } \\
\text { c }\end{array}$ & $\begin{array}{c}18.90 \\
833\end{array}$ & $\begin{array}{c}19.45 \\
833\end{array}$ & $\begin{array}{c}18.80 \\
833\end{array}$ & $\begin{array}{c}17.69 \\
167\end{array}$ & $\begin{array}{c}18.35 \\
833\end{array}$ & $\begin{array}{c}16.37 \\
5\end{array}$ & $\begin{array}{c}13.46 \\
667\end{array}$ & $\begin{array}{c}11.23 \\
333\end{array}$ & 9.575 & $\begin{array}{c}12.11 \\
667\end{array}$ & 14.5 & $\begin{array}{c}13.68 \\
333\end{array}$ & $\begin{array}{c}13.95 \\
833\end{array}$ \\
\hline Slovenia & $\begin{array}{c}6.741 \\
667\end{array}$ & $\begin{array}{c}6.191 \\
667\end{array}$ & $\begin{array}{c}6.341 \\
667\end{array}$ & 6.7 & $\begin{array}{c}6.341 \\
667\end{array}$ & $\begin{array}{c}6.541 \\
667\end{array}$ & $\begin{array}{c}5.991 \\
667\end{array}$ & $\begin{array}{c}4.858 \\
333\end{array}$ & $\begin{array}{c}4.391 \\
667\end{array}$ & $\begin{array}{c}5.891 \\
667\end{array}$ & 7.275 & $\begin{array}{c}8.208 \\
333\end{array}$ & $\begin{array}{c}8.891 \\
666\end{array}$ \\
\hline Spain & $\begin{array}{c}11.91 \\
667\end{array}$ & $\begin{array}{c}10.55 \\
833\end{array}$ & $\begin{array}{c}11.44 \\
167\end{array}$ & $\begin{array}{c}11.49 \\
167\end{array}$ & $\begin{array}{c}10.95 \\
833\end{array}$ & $\begin{array}{c}9.166 \\
667\end{array}$ & $\begin{array}{c}8.458 \\
333\end{array}$ & 8.225 & $\begin{array}{c}11.26 \\
667\end{array}$ & $\begin{array}{c}17.86 \\
667\end{array}$ & $\begin{array}{c}19.87 \\
5\end{array}$ & $\begin{array}{c}21.40 \\
833\end{array}$ & $\begin{array}{c}24.79 \\
167\end{array}$ \\
\hline Sweden & 5.6 & 5.825 & 5.95 & $\begin{array}{c}6.566 \\
667\end{array}$ & 7.375 & $\begin{array}{c}7.641 \\
667\end{array}$ & $\begin{array}{c}7.041 \\
667\end{array}$ & $\begin{array}{c}6.116 \\
667\end{array}$ & $\begin{array}{c}6.166 \\
667\end{array}$ & 8.3 & 8.575 & $\begin{array}{c}7.766 \\
667\end{array}$ & $\begin{array}{c}7.966 \\
667\end{array}$ \\
\hline $\begin{array}{l}\text { United } \\
\text { Kingdo } \\
\text { m }\end{array}$ & $\begin{array}{c}5.458 \\
603\end{array}$ & $\begin{array}{c}5.096 \\
97\end{array}$ & $\begin{array}{c}5.184 \\
958\end{array}$ & $\begin{array}{c}5.007 \\
329\end{array}$ & $\begin{array}{c}4.747 \\
302\end{array}$ & $\begin{array}{c}4.823 \\
375\end{array}$ & $\begin{array}{c}5.417 \\
598\end{array}$ & $\begin{array}{c}5.329 \\
14\end{array}$ & $\begin{array}{c}5.681 \\
96\end{array}$ & $\begin{array}{c}7.611 \\
054\end{array}$ & $\begin{array}{c}7.867 \\
382\end{array}$ & $\begin{array}{c}8.106 \\
182\end{array}$ & $\begin{array}{c}7.965 \\
578 \\
\end{array}$ \\
\hline $\begin{array}{l}\text { United } \\
\text { States }\end{array}$ & $\begin{array}{c}3.991 \\
667 \\
\end{array}$ & $\begin{array}{c}4.733 \\
333\end{array}$ & 5.775 & $\begin{array}{c}5.991 \\
667\end{array}$ & $\begin{array}{c}5.533 \\
333 \\
\end{array}$ & $\begin{array}{c}5.066 \\
667\end{array}$ & $\begin{array}{c}4.616 \\
667 \\
\end{array}$ & $\begin{array}{c}4.616 \\
667\end{array}$ & 5.775 & $\begin{array}{c}9.266 \\
666\end{array}$ & $\begin{array}{c}9.616 \\
667\end{array}$ & 8.95 & $\begin{array}{c}8.066 \\
667\end{array}$ \\
\hline
\end{tabular}

\begin{tabular}{|c|c|c|c|c|c|c|c|}
\hline & 2013 & 2014 & 2015 & 2016 & 2017 & 2018 & 2019 \\
\hline & & & & & & & \\
\hline Australia & 5.66194 & 6.077244 & 6.056423 & 5.710301 & 5.59376 & 5.299855 & 5.159245 \\
\hline Austria & 5.358333 & 5.641667 & 5.733333 & 6.033333 & 5.516667 & 4.858333 & 4.508333 \\
\hline Belgium & 8.45 & 8.533334 & 8.5 & 7.858333 & 7.1 & 5.958333 & 5.366667 \\
\hline Canada & 7.125 & 6.941667 & 6.941667 & 7.05 & 6.4 & 5.891667 & 5.733333 \\
\hline Chile & 6.08222 & 6.495096 & 6.328377 & 6.684913 & 6.965175 & 7.376639 & 7.22264 \\
\hline $\begin{array}{c}\text { Czech } \\
\text { Republic }\end{array}$ & 6.966667 & 6.116667 & 5.058333 & 3.966667 & 2.908333 & 2.266667 & 2.016667 \\
\hline Denmark & 7.391667 & 6.883333 & 6.3 & 6.008333 & 5.816667 & 5.116667 & 5.058333 \\
\hline
\end{tabular}




\begin{tabular}{|c|c|c|c|c|c|c|c|}
\hline Estonia & 8.566667 & 7.383333 & 6.208333 & 6.766667 & 5.816667 & 5.375 & 4.4 \\
\hline Finland & 8.191667 & 8.658334 & 9.375 & 8.791667 & 8.633333 & 7.358333 & 6.691667 \\
\hline France & 10.31667 & 10.3 & 10.35833 & 10.04167 & 9.425 & 9.025 & 8.45 \\
\hline Germany & 5.241667 & 4.991667 & 4.633333 & 4.133333 & 3.758333 & 3.4 & 3.15 \\
\hline Greece & 27.49167 & 26.55 & 24.95833 & 23.56667 & 21.53333 & 19.30833 & 17.33333 \\
\hline Hungary & 9.85 & 7.5 & 6.633333 & 4.975 & 4.05 & 3.591667 & 3.3 \\
\hline Iceland & 3.341667 & 3.283333 & 3.1 & 3.933333 & & & \\
\hline Ireland & 13.78333 & 11.91667 & 9.958333 & 8.408334 & 6.741667 & 5.766667 & 4.966667 \\
\hline Israel & 6.208333 & 5.908333 & 5.241667 & 4.808333 & 4.216667 & 4 & 3.8 \\
\hline Italy & 12.13333 & 12.65 & 11.89167 & 11.675 & 11.225 & 10.61667 & 9.966666 \\
\hline Japan & 4.025 & 3.591667 & 3.375 & 3.116667 & 2.808333 & 2.441667 & 2.35 \\
\hline South Korea & 3.1 & 3.491667 & 3.591667 & 3.675 & 3.683333 & 3.833333 & 3.783333 \\
\hline Latvia & 11.875 & 10.85 & 9.883333 & 9.633333 & 8.708333 & 7.4 & 6.308333 \\
\hline Lithuania & 11.8 & 10.725 & 9.133333 & 7.9 & 7.116667 & 6.183333 & 6.283333 \\
\hline Luxembourg & 5.875 & 6.05 & 6.458333 & 6.333333 & 5.616667 & 5.5 & 5.591667 \\
\hline Mexico & 4.929466 & 4.828958 & 4.352943 & 3.881356 & 3.419046 & 3.328549 & 3.49458 \\
\hline Netherlands & 7.241667 & 7.425 & 6.891667 & 6.025 & 4.858333 & 3.841667 & 3.4 \\
\hline New Zealand & 5.85 & 5.425 & 5.4 & 5.125 & 4.725 & 4.325 & 4.1 \\
\hline Norway & 3.758333 & 3.633333 & 4.5 & 4.758333 & 4.191667 & 3.875 & 3.7 \\
\hline Poland & 10.34167 & 9.016666 & 7.525 & 6.191667 & 4.883333 & 3.858333 & 3.283333 \\
\hline Portugal & 16.45833 & 14.125 & 12.65833 & 11.175 & 9.016666 & 7.05 & 6.55 \\
\hline $\begin{array}{c}\text { Slovak } \\
\text { Republic } \\
\end{array}$ & 14.225 & 13.20833 & 11.5 & 9.666667 & 8.125 & 6.541667 & 5.766667 \\
\hline Slovenia & 10.15833 & 9.741667 & 9 & 8.008333 & 6.6 & 5.125 & 4.45 \\
\hline Spain & 26.11667 & 24.45 & 22.075 & 19.65 & 17.23333 & 15.26667 & 14.10833 \\
\hline Sweden & 8 & 7.933333 & 7.4 & 6.95 & 6.683333 & 6.325 & 6.766667 \\
\hline $\begin{array}{c}\text { United } \\
\text { Kingdom }\end{array}$ & 7.603673 & 6.178483 & 5.383935 & 4.892704 & 4.401906 & 4.080814 & 3.833521 \\
\hline United States & 7.375 & 6.166667 & 5.291667 & 4.866667 & 4.35 & 3.9 & 3.666667 \\
\hline
\end{tabular}

Source: OECD, GDP Database: https://stats.oecd.org/

Table $3 b$

Average annual unemployment rate of OECD countries.

\begin{tabular}{|c|c|c|c|c|c|c|c|c|c|c|c|c|c|}
\hline Year & 2000 & 2001 & 2002 & 2003 & 2004 & 2005 & 2006 & 2007 & 2008 & 2009 & 2010 & 2011 & 2012 \\
\hline $\begin{array}{c}\text { Unemployme } \\
\text { nt Rate (\%) }\end{array}$ & $\begin{array}{c}6.78 \\
\%\end{array}$ & $\begin{array}{c}6.62 \\
\%\end{array}$ & $\begin{array}{c}7.18 \\
\%\end{array}$ & $\begin{array}{c}7.30 \\
\%\end{array}$ & $\begin{array}{c}7.19 \\
\%\end{array}$ & $\begin{array}{c}6.83 \\
\%\end{array}$ & $\begin{array}{c}6.27 \\
\%\end{array}$ & $\begin{array}{c}5.81 \\
\%\end{array}$ & $\begin{array}{c}6.14 \\
\%\end{array}$ & $\begin{array}{c}8.28 \\
\%\end{array}$ & $\begin{array}{c}8.43 \\
\%\end{array}$ & $\begin{array}{c}8.03 \\
\%\end{array}$ & $\begin{array}{c}8.01 \\
\%\end{array}$ \\
\hline $\begin{array}{c}\text { Annual } \\
\text { Change }\end{array}$ & $\begin{array}{c}0.39 \\
\%\end{array}$ & $\begin{array}{c}0.15 \\
\%\end{array}$ & $\begin{array}{c}0.56 \\
\%\end{array}$ & $\begin{array}{c}0.12 \\
\%\end{array}$ & $\begin{array}{c}0.11 \\
\%\end{array}$ & $\begin{array}{c}0.36 \\
\%\end{array}$ & $\begin{array}{c}0.56 \\
\%\end{array}$ & $\begin{array}{c}0.45 \\
\%\end{array}$ & $\begin{array}{c}0.32 \\
\%\end{array}$ & $\begin{array}{c}2.14 \\
\%\end{array}$ & $\begin{array}{c}0.15 \\
\%\end{array}$ & $\begin{array}{c}0.40 \\
\%\end{array}$ & $\begin{array}{c}0.02 \\
\%\end{array}$ \\
\hline
\end{tabular}




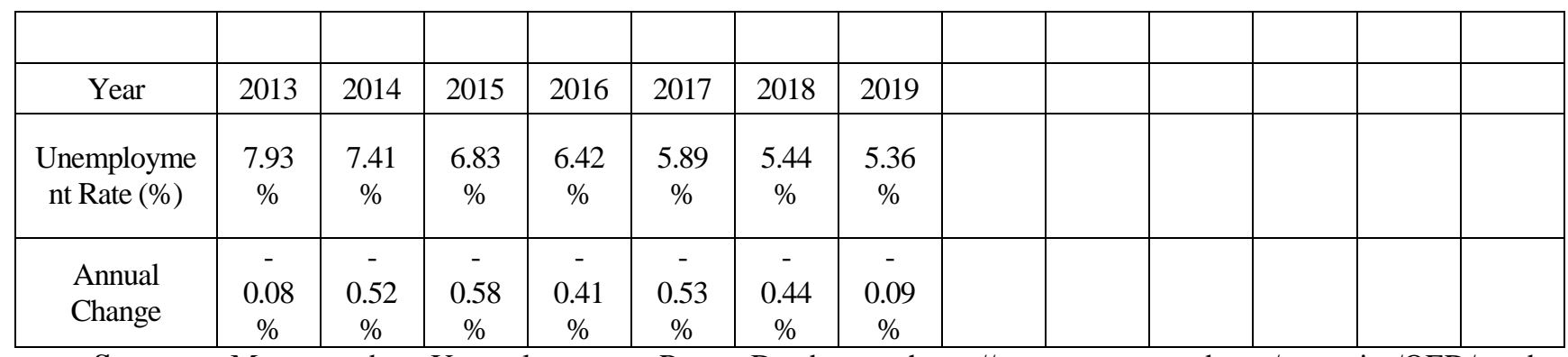

Source: Macrotrends, Unemployment Rate Database: https://www.macrotrends.net/countries/OED/oecdmembers/unemployment-rate

Table 4

Correlations

\section{Correlation Analysis between Tax and GDP.}

\begin{tabular}{|c|c|c|c|}
\hline & Tax & GDP \\
\hline Tax & Pearson Correlation & 1 & .256 \\
& Sig. (2-tailed) & & .275 \\
GDP & $\mathrm{N}$ & 20 & 20 \\
& Pearson Correlation & .256 & 1 \\
& Sig. (2-tailed) & .275 & 20 \\
$\mathrm{~N}$ & 20 & \\
\hline
\end{tabular}

Table 5

Correlations

\section{Correlation Analysis between Tax and Unemployment.}

\begin{tabular}{|c|c|c|c|}
\hline & & Tax & Unemployment \\
\hline \multirow{6}{*}{ Unemployment } & Pearson Correlation & 1 & .086 \\
\hline & Sig. (2-tailed) & & .718 \\
\hline & $\mathrm{N}$ & 20 & 20 \\
\hline & Pearson Correlation & .086 & 1 \\
\hline & Sig. (2-tailed) & .718 & \\
\hline & $\mathrm{N}$ & 20 & 20 \\
\hline
\end{tabular}

